\title{
Differentiation of cortical brain organoids and optic nerve-like structures from retinal confluent cultures of pluripotent stem cells.
}

1 Milan Fernando ${ }^{1, \#}$, Scott Lee ${ }^{1, \#}$, Jesse R. Wark ${ }^{1,2}$, Di Xiao ${ }^{3,4,5}$, Hani J. Kim ${ }^{3,4,5}$, Grady C. Smith ${ }^{1}$, Ted 2 Wong $^{6}$, Erdahl T. Teber ${ }^{5,6}$, Robin R. Ali ${ }^{7,8}$, Pengyi Yang ${ }^{3,4,5}$, Mark E. Graham ${ }^{2,5}$, Anai Gonzalez3 Cordero ${ }^{1,5, *}$.

4

5 'Stem Cell Medicine Group, ${ }^{2}$ Synapse Proteomics, ${ }^{3}$ Computational Systems Biology Group, 6 BBioinformatics; Children's Medical Research Institute, University of Sydney, Westmead, 2145, NSW, 7 Australia; ${ }^{4}$ Charles Perkins Centre, School of Mathematics and Statistics, University of Sydney, Sydney, NSW 2006, Australia; ${ }^{5}$ School of Medical Sciences, Faculty of Medicine and Health, University of Sydney, NSW 2006; ' Gene and Cell Therapy Group, UCL Institute of Ophthalmology, London EC1V 9EL, UK. ${ }^{8}$ Current address: Centre for Cell and Gene Therapy, King's College London, 8th Floor Tower Wing, Guy's Hospital, London SE1 9RT, UK.

\#these two authors contributed equally to the work

*Corresponding author: Dr Anai Gonzalez Cordero

agonzalez-cordero@cmri.org.au

Stem Cell Medicine Group, Children's Medical Research Institute and University of Sydney 214 Hawkesbury Road, Westmead, 2145, NSW, Australia

Tel: $+61(2) 88652980$

Running title: Cortical brain organoids from confluent iPS cell cultures

Key worlds: brain organoids, retinal organoids, pluripotent stem cells, omics, multi electrode array, 


\section{Abstract}

Advances in the study of neurological conditions have been possible due to induced pluripotent stem cell technologies and the generation of neural cell types and organoids. Numerous studies have described the generation of neural ectoderm-derived retinal and brain structures from pluripotent stem cells. However, the field is still troubled by technical challenges, including high culture costs and organoid-to-organoid variability. Here, we describe a simple and economical protocol that reproducibly gives rise to the neural retina and cortical brain regions from confluent cultures of stem cells. The spontaneously generated cortical organoids were isolated and cultured in suspension conditions for maturation and are transcriptionally comparable to organoids generated by other methods and to human foetal cortex. Furthermore, these organoids show spontaneous functional network activity with proteomic analysis and electron microscopy demonstrating the presence of synaptic components and maturity. The generation of retinal and brain organoids in close proximity also enabled their mutual isolation. Further culture of this complex organoid system demonstrated the formation of optic nervelike structures connecting retinal and brain organoids, which might facilitate the investigation of the mechanisms of neurological diseases of the eye and brain.

\section{Introduction}

The rapidly progressing field of human pluripotent stem cells (hPSCs), including embryonic (ES) and induced pluripotent stem (iPS) cells, and their derivative organoids continue to provide new insights into basic biology, human development, modelling of human diseases and discovery of innovative treatments. Neural differentiation, in particular, has been extensively studied, improving our understanding of the mechanism of neurodevelopmental conditions ${ }^{1,2,3}$. Large numbers of neurons and astrocytes are able to be generated using two-dimensional (2D) cultures derived from hPSCs ${ }^{4,5}$ and these classical differentiation cultures were later optimised to three-dimensional (3D) suspension methods that better recapitulate the physiological niche and environment of the developing human brain ${ }^{6}$.

In the developing central nervous system (CNS), the eye and the brain form as an extension of the forebrain diencephalic and telencephalic region, respectively ${ }^{7}$. Developmental biology studies have helped infer the molecular basis of this patterning process and facilitated the establishment of numerous differentiation protocols, which generate miniaturised versions of 3D organoids from PSCs. Brain organoids replicate specific brain regions or whole cerebral areas, with both occasionally developing eye regions ${ }^{8} 9,10$. 
Differentiation protocols are usually classified as either guided/direct or non-guided/undirected cultures, based on the necessity for guided differentiation using growth factors or their absence in non-guided spontaneous differentiation, which relies on endogenous self-forming ability of the cells ${ }^{11}$. Brain organoids generated using these varied methodologies have been comprehensively characterised using transcriptome analysis by RNA sequencing. Single cell RNA sequencing (scRNA-seq) has elucidated the cellular composition of organoids and reproducibility of organoid protocols ${ }^{12,13,1,10,14-16}$. However, the differentiation of PSC-derived brain organoids presents challenges, such as variability within and between organoid batches and high culture costs. This lack of reproducibility demonstrates the necessity of novel differentiation approaches. Furthermore, unbiased omics, including proteome studies, together with robust measurements of neuronal activity, are critical to establish organoid variability, maturation and functionality.

Diseases of the eye and the brain are now understood to be more intertwined than previously thought. Studies of common conditions, such as Alzheimer's disease and glaucoma have demonstrated neurodegenerative changes and disease traits in both brain and eye regions ${ }^{17,18}$. Complex organoids have the potential to provide useful models of these human disease in vitro with the proviso that they faithfully recapitulate retinal development, morphology and maturation. Therefore, improved formation of hPSC-derived retinal-brain connection through an optic nerve is essential for effective degenerative disease modelling.

Here we hypothesised that it is possible to reproducibly generate functional brain organoids from retinal confluent cultures of PSCs. We also asked the question if the retinal and brain organoids developing in suspension culture together would form a complex retina-brain organoid system that would enable the formation of an optic nerve-like structure.

We demonstrated here that the non-guided, simple and economical differentiation protocol, previously described to spontaneously generate retinal vesicles from a confluent culture of hPSCs ${ }^{19-21}$, also generated brain organoids alongside the differentiation of retinal vesicles. The ease of precisely locating cortical organoids, due to morphology and their proximity to retinal vesicles, reduced organoid variability. Brain organoids were characterised as dorsal cortical organoids which, when further cultured in 3D suspension, matured into functional organoids. A systematic comparison of scRNA-seq datasets revealed a close similarity of our organoids with other dorsal patterned hPSC-derived brain organoids ${ }^{16}$ and proteomic analysis of late-stage organoids revealed the presence of numerous synaptic markers. Organoids electrophysiological activity was dependent on organoids being cultured in relevant basal medium $^{22}$ with proteomics providing insights into why this physiological environment aids 
neurophysiological activity. Finally, the generation of retinal and cortical organoids facilitated the isolation of both structures for 3D culturing, forming a complex organoid system. Notably, when cultured in suspension, these retinal-brain organoids maintained the natural association created in the dish during their spontaneous development which enabled the formation of optic nerve-like structures between the two organoids.

\section{Results}

\section{Generation of self-forming retinal and dorsal cortical brain organoids from Confluent 2D/3D cultures}

The retina is an extension of the CNS arising from the forebrain region in the developing embryo (Figure 1a). Indeed, the differentiation of whole brain cerebral organoids containing retinal structures has been demonstrated $^{8,10}$. Figure 1b schematic shows that proneural induction of confluent cultures of PSCs spontaneously generate pigmented islands of retinal pigment epithelium (RPE) from which retinal vesicles appear ${ }^{19,21}$. Further analysis of other structures forming in this 2D/3D environment highlighted the presence of $3 \mathrm{D}$ regions containing clear neuronal rosettes forming in close proximity to retinal vesicles. Based on the neuronal culture environment, and their morphological characteristics, we hypothesised that these were brain vesicles. To fully characterise these structures, we manually excised these regions to be grown in $3 \mathrm{D}$ suspension for maturation in culture, as previously described for their retinal counterparts ${ }^{21}$.

Following a neural induction period of 4-6 weeks in culture, neuroretinal vesicles and neural rosette structures appeared (Figure 1c). These areas were manually dissected and grown in suspension in retinal media from 7 weeks onwards to allow for maturation in a media composition previously described in Lancaster et al., 2013 (Figure 1d). By 6 weeks of differentiation, 39\% of all organoids observed in cultures were retinal vesicles while $60 \%$ were neuronal organoids ( $n=102 D / 3 \mathrm{D}$ differentiation batches) demonstrating that a proportion of cortical organoids are generated independently and are not in proximity to retinal organoids. Immunohistochemistry (IHC) of 6-week old organoids revealed highly proliferative KI67/NCAD positive neuroepithelium regions (Figure 1e). Active CASPASE3 cells were also observed in the organoids. To mitigate this phenotype, organoids were further cultured on previously-described shaking platforms (Figure 1f). Well-formed neuroepithelium regions contained numerous SOX2/NCAD positive neural progenitor cells (Figure 19, $\mathbf{h}$ ) that were also immuno-positive for cortical FOXG1 and NESTIN/PAX6 markers (Figure 1j, S1a and SMovie 1). These cortical organoids were negative for ventral cortex specific marker, NKX2.1 (Figure S1b), as opposed to whole brain cerebral organoids (Figure S1c), confirming the dorsal cortical origin of these organoids. 
133 The presence of RPE and typical retinal vesicles facilitated the separation of dorsal cortical brain organoids under bright-field microscopy from other forebrain-like neuroepithelia which also form in these cultures (Figure S1d-f). These dorsal brain organoids could be easily distinguished in the 2D/3D confluent cultures enabling the isolation of a population of dorsal cortical organoids for further maturation and therefore minimising variability of mature cultures. We observed successful differentiation and these culture features across a number of iPS stem cell lines (Figure S2).
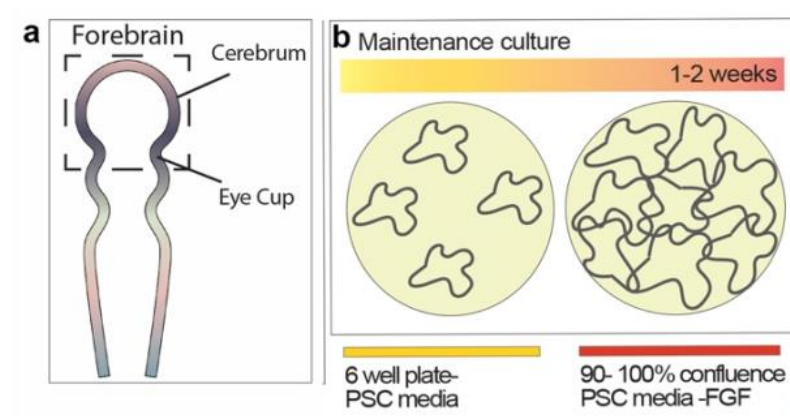

$$
\begin{aligned}
& N \\
& 6 \\
& 6
\end{aligned}
$$

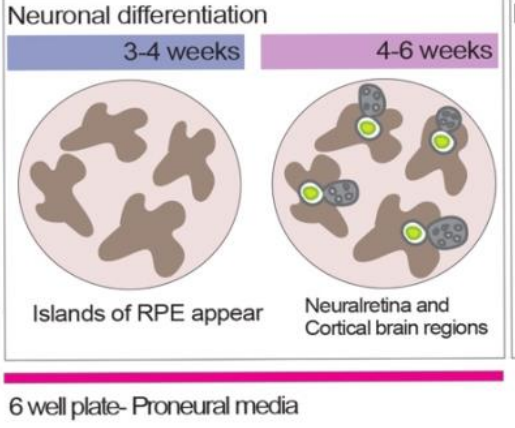

Neuronal maturation 4-20+ weeks
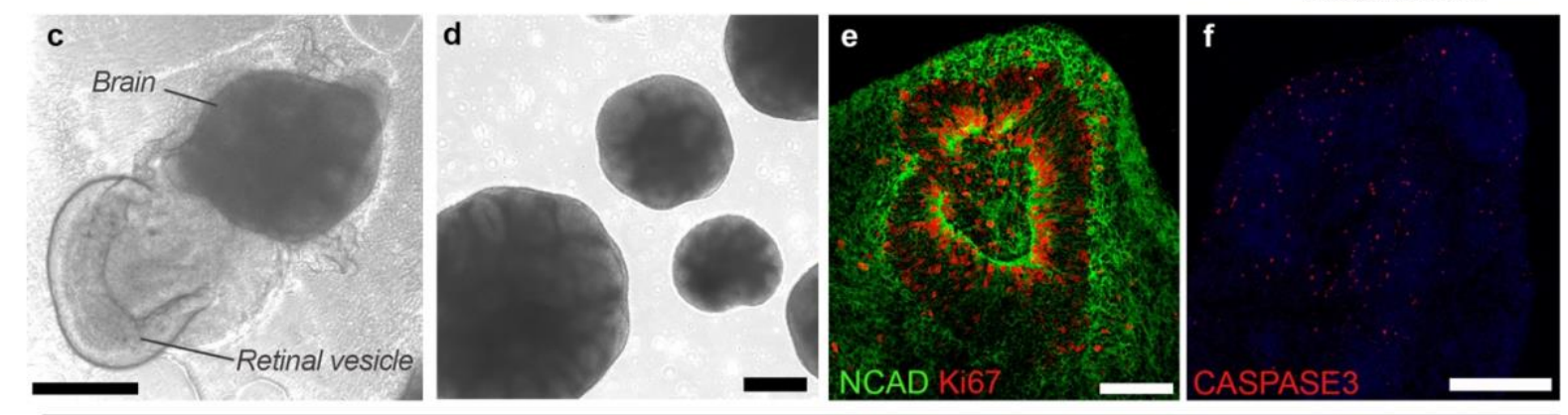

Dorsal Cortical Brain organoids
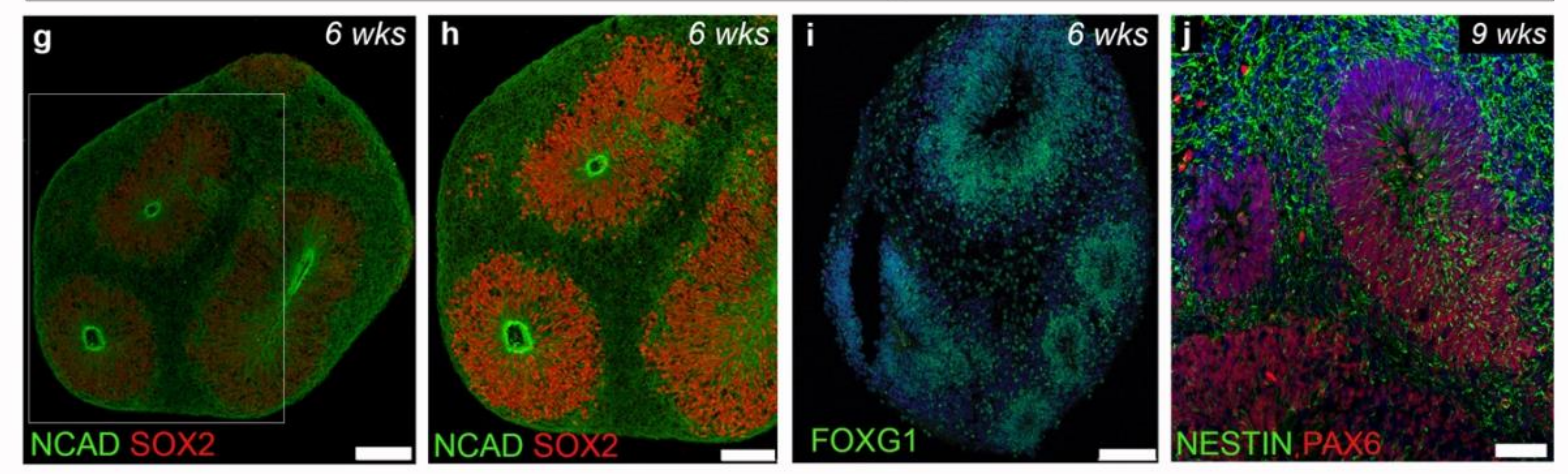
Schematic illustrating neural tube formation and the formation of both cerebellum and eye cups from forebrain. b. Schematic summarising the 2D/3D differentiation protocol timeline for both retinal and cortical organoids formation as well as media composition and maturation in suspension. c. Representative image of a retinal vesicle and brain organoid in 2D culture. d. Bright-field image showing floating brain organoids, with typical neural rosettes, following manually excision from 2D culture. e-j. Immunohistochemistry images of 6 -9 weeks old brain organoids. e, f. Brain organoids showing Ki67 proliferative NCAD positive neuroepithelium and CASPASE 3 apoptotic cells. $\mathbf{g}$-j. Cortical origin of brain organoids is confirmed by the presence of SOX2, FOXG1 and PAX6 positive neural precursors markers. 


\section{Cortical organoids differentiate into Cortical neurons and Glial cell types}

152 Next, IHC confirmed the presence of cortical plate markers TBR1 which co-expressed with CTIP2-

153 positive cells in the cortical organoids (Figure 2a-c). By 10 weeks of culture, CTIP2 regions also 154 contained SATB2-positive cells (Figure 2e, f). From 8 to 12 weeks in culture, organoids increased in 155 size significantly from $2000 \mu \mathrm{m}( \pm 560)$ to $2600 \mu \mathrm{m}( \pm 474)$ in diameter (Figure $\mathbf{2 g}, \mathrm{n}=20-25$ organoids, $156 \mathrm{~N}=3$ differentiation batches; mean $\pm \mathrm{SD}, \mathrm{p}=0.0002$, unpaired two-tailed $t$ test). This differentiation 157 protocol also supported the differentiation of S100B- (Figure 2g) and GFAP-positive astrocytes that 158 were present in similar percentages to TUJ1-positive neurons (Figure $\mathbf{2} \mathbf{h}, \mathbf{i}, \mathbf{7 5} \%( \pm 14 \%$ ) neurons versus $15957 \%( \pm 17 \%)$ glial cells; $n=10$ images; $N=3-4$ differentiation batches; mean $\pm S D, p<0.0001$, unpaired 160 two-tailed $t$ test). Further IHC analysis at 15 weeks ( 3.5 months) in culture demonstrated the presence 161 of more mature inhibitory gamma aminobutyric acid (GABAergic) neurons (Figure 2j, k (high 162 magnification of boxed area in A)) and their CALRETININ-positive subtypes (Figure 2l). Furthermore, 163 3D imaging of the whole-brain organoid by Lightsheet microscopy confirmed the presence of numerous 164 inhibitory CALRETININ-positive neurons and GFAP-positive astroglial cell types (SMovie 2).
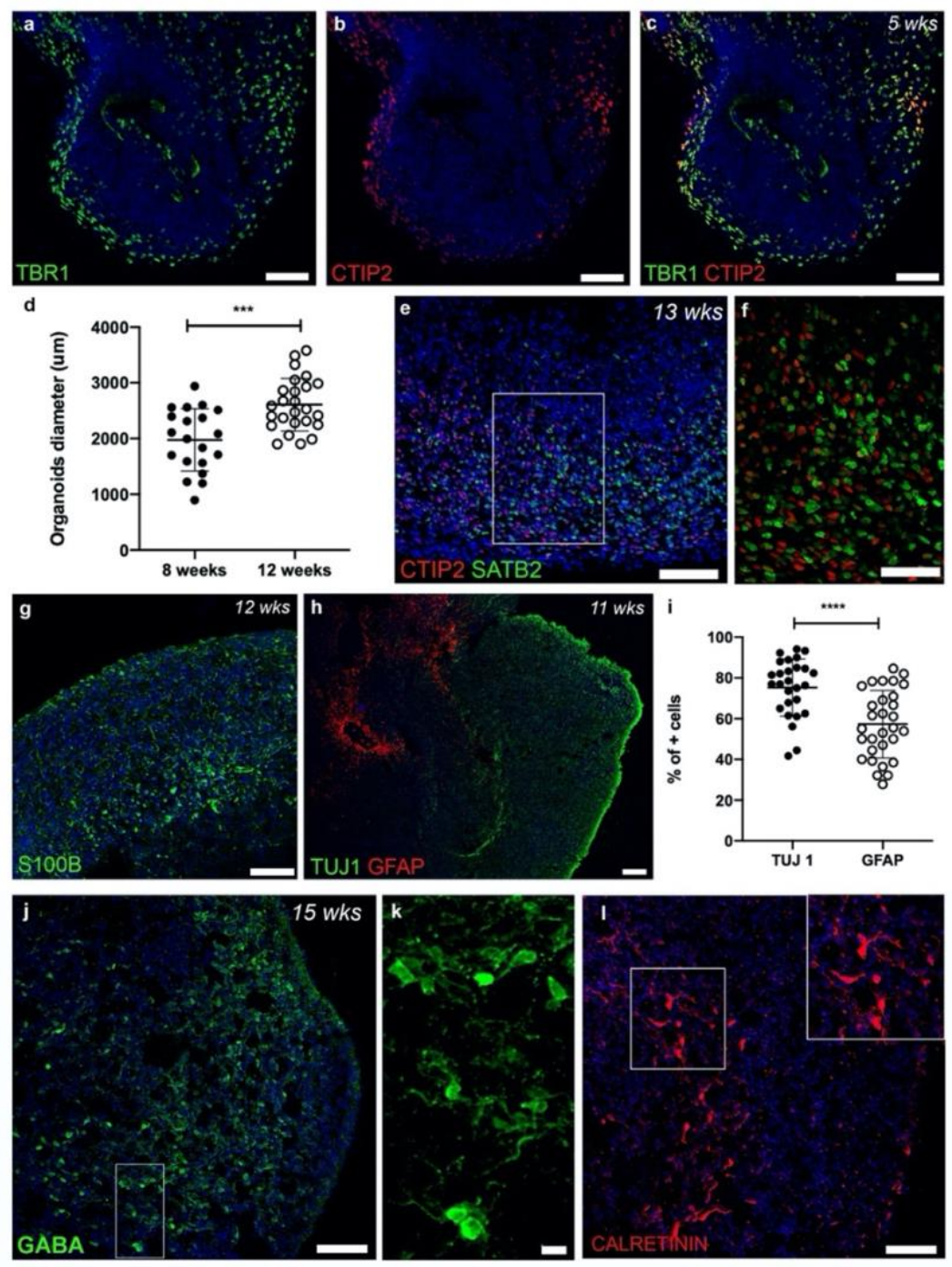
166 Figure 2. Cortical brain organoids generate cortical plate neurons and glial cells. a-c.

167 Representative immunohistochemistry images of week 5 cortical organoids showing the presence of

168 TBR1 and CTIP2 cortical plate neurons. d. Graph showing cortical organoids diameter and their

169 significant size increase with days in culture $(n=30$ organoids, $N=3$ differentiation batches, mean $\pm S D$,

170 unpaired two-tailed $t$ test). e-h. Representative images of organoids showing CTIP2 and SATB2 positive

171 cells $(\mathbf{e}, \mathbf{f}$; high magnification image of inset in $\mathbf{e}$ shown in $\mathbf{f})$ and S100B and GFAP glial cells $(\mathbf{g}, \mathbf{h})$. i.

172 Graph showing the percentage of cells expressing TUJ1 and GFAP per area (mm2) in 11 weeks old

173 cells. ( $n=10$ images from 2-3 organoids, $N=3$ differentiation batches, mean $\pm S D$, unpaired two-tailed

$174 t$ test). j-I. Week 15 cortical organoid showing GABA and CALRETININ positive inhibitory neurons, $\mathbf{k}$

175 shows high magnification image of inset in j. Scale bars, $15 \mu \mathrm{m}(\mathrm{k}), 50 \mu \mathrm{m}$ (f), $75 \mu \mathrm{m}(\mathrm{a}-\mathrm{c}), 100 \mu \mathrm{m}(\mathrm{e}$,

$176 \mathrm{~g}, \mathrm{~h}, \mathrm{j}, \mathrm{l})$.

177

178 Cortical Organoids from 2D/3D confluent cultures have similar cell type compositions to other 179 brain organoids

180 Next, we performed scRNA-seq analysis using the 10X genomics platform to further investigate the cell-

181 type composition of these dorsal cortical organoids and to establish their similarity to other published

182 brain organoid scRNA-seq datasets, which were derived from various directed and undirected protocols.

183 We used scClassify ${ }^{23}$, a machine learning-based method, to annotate cell types that were present in our

184 scRNA-seq cortical organoids (Figure 3a) and compared the composition of cell types in 3-month-old

185 cortical organoid (CO) we generated against those from 3- and 6-month-old brain organoids and cell

186 types from gestational week 12 (GW12) pre-frontal lobe (PFL) foetal brain (Figure 3b).

187

188 First, we compared the composition of cell types by using the cell type labels from the original authors

189 for each of the published datasets, summarised in the table in Figure $\mathbf{3 b}$. Although the proportions of

190 each cell type varied across protocols, the overall cell type compositions were similar. This was

191 independent of the protocol, their method of differentiation (directed or undirected) and age of the

192 organoid (Figure 3c). In this analysis, Quadrato et al data (Quad 3M U) showed the most diverse cell-

193 type composition.

195 Next, we utilised scClassify to annotate cells in our CO scRNA-seq dataset by training the classification

196 model using either each individual public dataset as a reference or using all datasets jointly (refer to as

197 joint training) (Figure 3d). The predicted cell-type composition in CO scRNA-seq data was plotted with

198 respect to the training data, and, irrespective of the training dataset, a similar cell-type composition was

199 observed in our COs (Figure 3d). In agreement with this, the expression profiles of key marker genes

200 for each cell type population were also largely consistent, irrespective of the training dataset (Figure

201 S3). BMP responsive cells, intermediate (bright orange) and proteoglycan-expressing cell types were absent from our dataset. Note that cells are annotated as "unassigned" when they could not be classified 203 to any cell type. Cells are annotated as "intermediate" when they could not be classified to a specific 204 cell type but an intermediate among multiple cell types. This intermediate population (denoted with “*”) 
205 is different from the intermediate cell type annotated in the training datasets (bright orange). Since the 206 scClassify jointly trained using multiple reference datasets reduces both unassigned and intermediate* 207 classification, the classification result from the jointly trained model was used in subsequent comparison. 208 Organoid cell types were clustered in a tSNE plot showing that cortical neurons were the most abundant 209 cell type (Figure 3e). We then measured the agreement between different cell-type compositions across 210 the multiple datasets using intraclass correlation (ICC) and visualised these results (Figure 3f). Three 211 major correlating groups were evident in relation to cell-type compositions: (1) Velasco et al., 6 mo., 212 directed (Vela 6m D); (2) Quadrato et al., 3 mo., undirected (Quad 3m U) and; (3) the remaining 213 protocols, which included our 2D/3D COs. In accordance with Figure 3c, except for Quad 3m U, all 3m 214 organoids and the GW12 foetal sample cluster together, suggesting similar cell-type composition. 215 Notably, our organoids closely resemble the guided differentiation of dorsally patterned forebrain 216 organoids (Vela 3M D), known for their reproducibility ${ }^{16}$. Cell-type proportions were more diverse when 217 Quad $3 \mathrm{~m} U$ was used as training data, suggesting a unique cell-type composition in this dataset. In fact, 218 Quad 3m U cell-type annotations clustered as a group with Vela 6m D separate from Trujillo et al., 6 219 mo., directed (Truj 6m D) and the remaining 3 mo. organoids. Therefore, whilst cell-type composition of 220 Vela $6 \mathrm{~m}$ D appeared to be different from those of $3 \mathrm{~m}$ organoids, the composition of Truj $6 \mathrm{~m}$ D was more 221 similar to $3 \mathrm{~m}$ organoids. These results demonstrated the transcriptional similarities between cortical 222 organoids from spontaneous 2D/3D cultures and previous described brain organoids and the foetal 223 nervous system. 


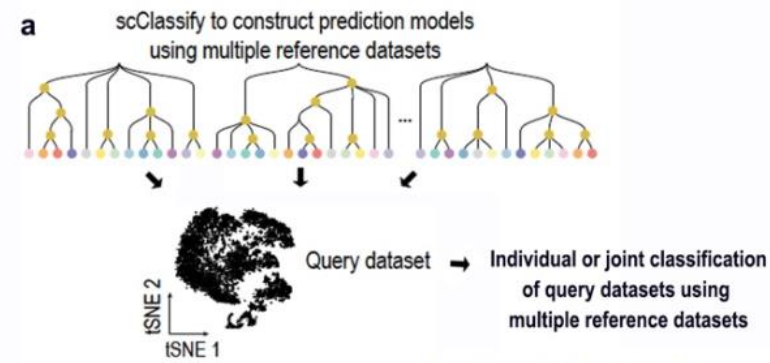

\begin{tabular}{|l|l|l|l|}
\hline \multicolumn{1}{|c|}{ Protocol } & Tissue/Organoid & \multicolumn{1}{c|}{ Age } & Method \\
\hline Zhong et al. & Brain (PFL) & GW12 & \\
\hline Giandomenico et al. & Whole brain org & 3 months & Undirect \\
\hline Birey et al. & Forebrain sphe & 3 months & Direct \\
\hline Velasco et al. & Dorsal CO org & 3 months & Direct \\
\hline Quadrato et al. & Whole brain org & 3 months & Undirect \\
\hline Trujillo et al. & Cortical org & 6 months & Direct \\
\hline Velasco et al. & Dorsal CO org & 6 months & Direct \\
\hline
\end{tabular}

c
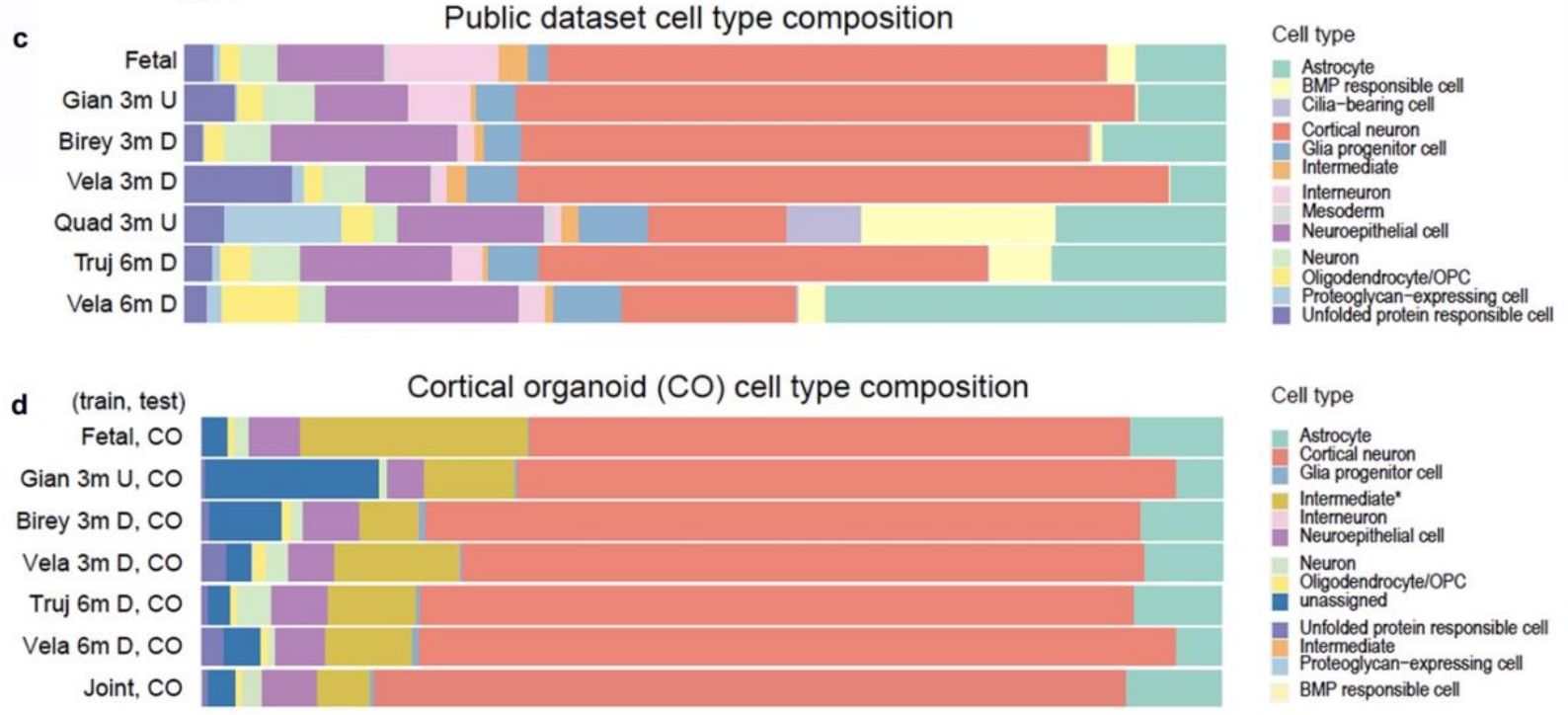

e
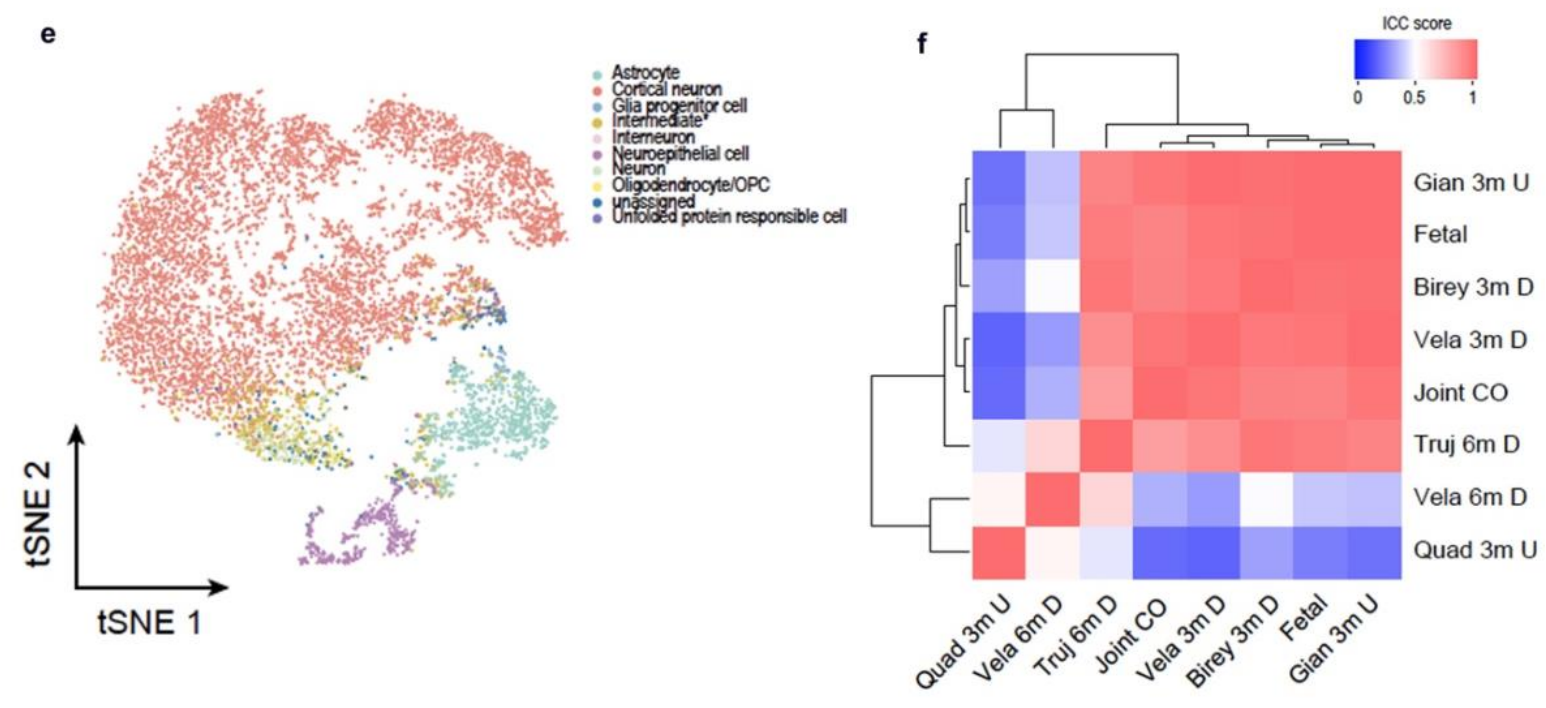

Figure 3. Comparison of cortical organoids and scRNA-seq available dataset. a. Schematic illustrating scClassify computational analysis to categorise a dataset using multiple reference datasets. b. Table summarising the public scRNA-seq reference datasets included in comparison and their differentiation protocols. c. Cell types composition in public datasets based on the annotation from their original studies. d. Cell-type composition in cortical organoids (CO) scRNA-seq dataset predicted by scClassify trained by each or all (i.e. Joint) public datasets. e. tSNE plot illustrating the cell types composition in $\mathrm{CO}$ based on scClassify annotation using Joint training. f. Interclass correlation heatmap showing the agreement between cell-type compositions among different organoids and a fetal brain. 
234 Proteome analysis of brain organoids highlights increased abundance of proteins related to 235 synaptic transmission

236 The proteomes of iPSCs and cortical organoids were surveyed to a depth of 6,244 and 5,719 proteins, 237 respectively (after filtering and counting only unique genes). There were 4,444 proteins present shared 238 between the iPSC and cortical organoid lists. Gene ontology enrichment analysis was performed with a 239 focus on biological process and KEGG pathway terms (Figure 4a-d). Figure 4a shows the top thirty 240 biological process terms that had the largest difference in significance between iPSCs and cortical 241 organoids. Terms related to the cell cycle, chromosomes and DNA were more likely to be enriched for 242 iPSCs, whereas terms related to the synapse, synaptic vesicles, vesical transport, axon/dendrite 243 development and neuron projection were more enriched for cortical organoids. Since we are interested 244 in neuronal differentiation in organoids, we extracted the top five synaptic and development terms (not 245 already shown in Figure 4a) from the list of significant biological process terms (Table S2). For example, 246 the terms "postsynapse organisation" and "regulation of postsynaptic membrane neurotransmitter 247 receptor levels" were enriched for cortical organoids (Figure 4b). Neuron development and dendritic 248 spine development were enriched for cortical organoids (Figure 4c). The KEGG pathway terms 249 ribosome biogenesis in eukaryotes, DNA replication, cell cycle, and basal transcription factors were 250 more enriched for iPSCs while axon guidance, neurotrophin signalling pathway and long-term 251 potentiation terms were enriched for cortical organoids (Figure 4d). Examples of detected proteins that 252 were exclusive to the iPSCs and are involved in stem cell proliferation and maintenance include proteins 253 encoded by LIN28A, BMPR1A, FGFR1, SOX2, ARID1A and RTF1 (Figure 4e). Conversely, GABA 254 receptor subunits (encoded by GABBR1 and GABRG2), a glutamate receptor subunit (encoded by 255 GRIA1), a mediator of postsynaptic plasticity (encoded by SYNGAP1), a trans-synaptic protein 256 (encoded by NRXN1) and two synaptic vesicle associated proteins (encoded by SYN1 and SYP) are 257 examples of proteins detected exclusively in cortical organoids (Figure 4e). The presence of these 258 proteins and enrichment of relevant gene ontology terms confirms there was strong proteomic evidence 259 that cortical organoids differentiated towards the neuronal- and away from a stem cell-phenotype. 
a

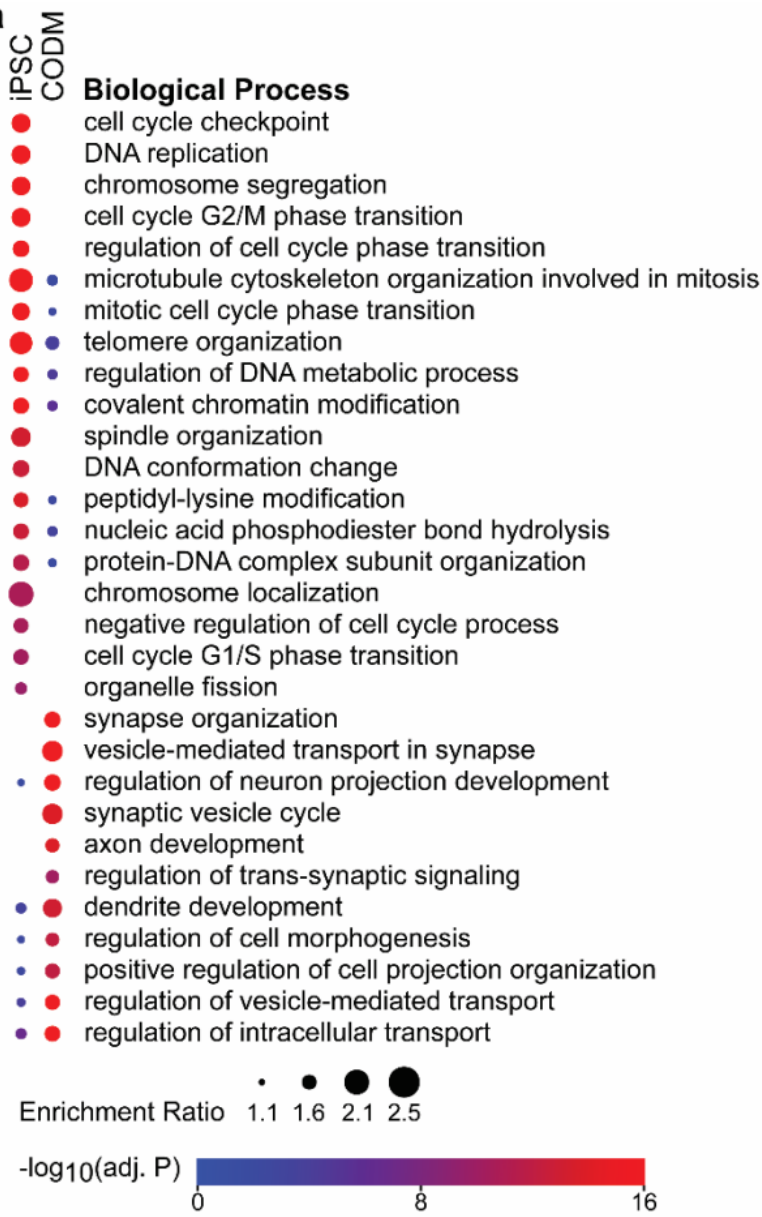

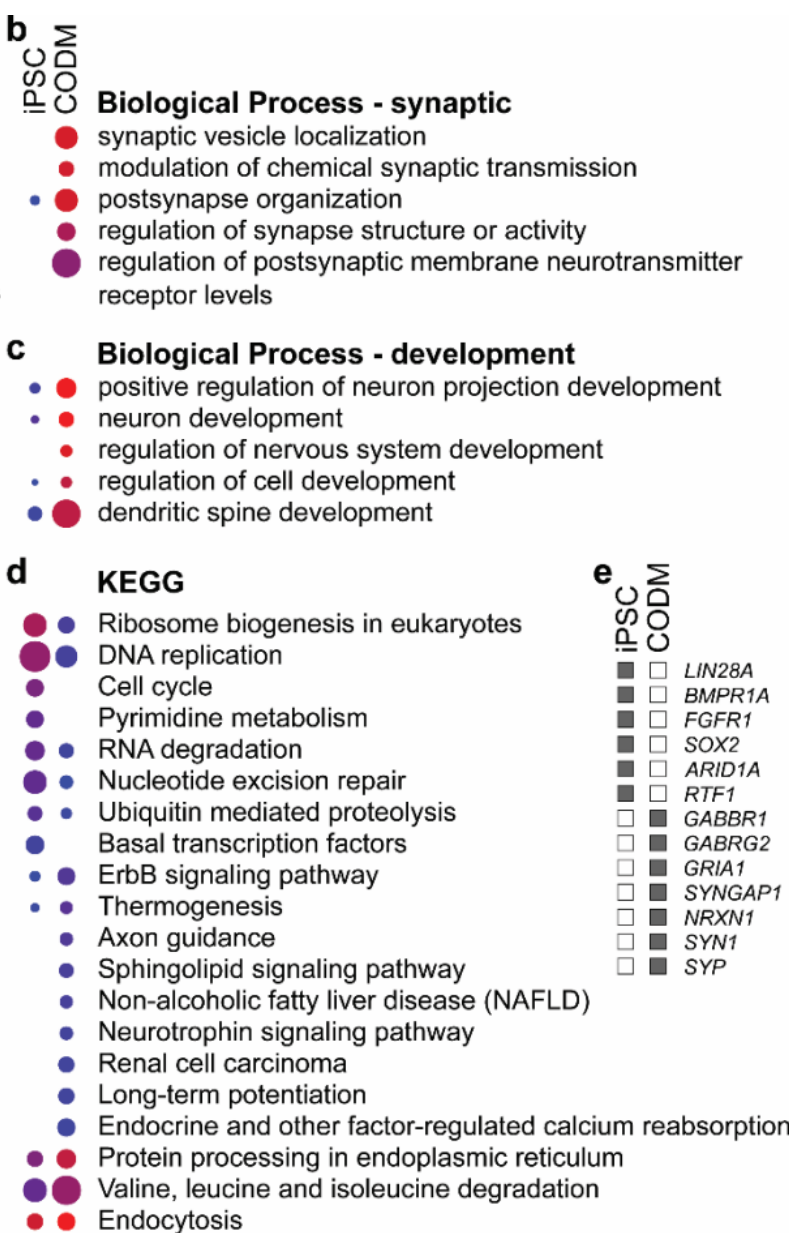

Figure 4. Comparison of the iPSC and cortical organoid proteomes using gene ontology enrichment analysis. a. Comparison of biological process terms with the largest difference in probability of enrichment for the genes encoding proteins detected in the iPSC and cortical organoid proteomes, the latter grown in cortical organoid differentiation medium (CODM). The colour scheme represents the value of $-\log _{10}(P)$, where $P$ is the probability of enrichment adjusted by a false discovery rate of $5 \%$. The size of each circle represents the ratio of enrichment. Absent circles indicate an enrichment ratio $<1.1$. b. The top five biological process synaptic terms not already shown in a. c. The top five biological process development terms not already shown in a. d. Comparison of the KEGG pathway terms. e. Examples of proteins detected exclusively in either iPSCs or cortical organoids involved in maintenance of stems cells or synaptic functions.

\section{D/3D iPSC-derived Cortical Brain Organoids showed mature neuronal morphology and}

\section{function.}

Next, we sought to evaluate maturation and functionality of cortical organoids. Maturation of neurons is usually attributed to the presence of synapses. We first used IHC to examine synapse formation in organoids. MAP2 positive glutamatergic excitatory neurons expressing the vesicular glutamate transporter 1 (VGLUT1) protein were evident in brain organoids (Figure 5a, b (high magnification of boxed area in A)). Pre-synaptic protein synaptophysin was readily detected in puncta that localised in proximity to postsynaptic density protein PSD95 (Figure 5c). Similarly, inhibitory GABA neurons and a 
280 punctate pattern of presynaptic VGLUT1 were also evident in 2D cultures of dissociated of brain 281 organoids (Figure S4). To comprehensively characterise the synaptic machinery in these organoids we 282 analysed the proteome of 5 months-old organoids ( $n=3$ organoids per batch, $N=3$ different batches of 283 differentiation): cell type specific enrichment confirmed the presence of all expected cell types including 284 neurons, astrocytes and oligodendrocytes. Finally, ultrastructure analysis by transmission electron 285 microscopy (TEM) demonstrated the presence of typical synaptic structures showing synaptic vesicles 286 and electron-dense synaptic contact sites (Figure 5d, e, asterisks and arrowheads, respectively).
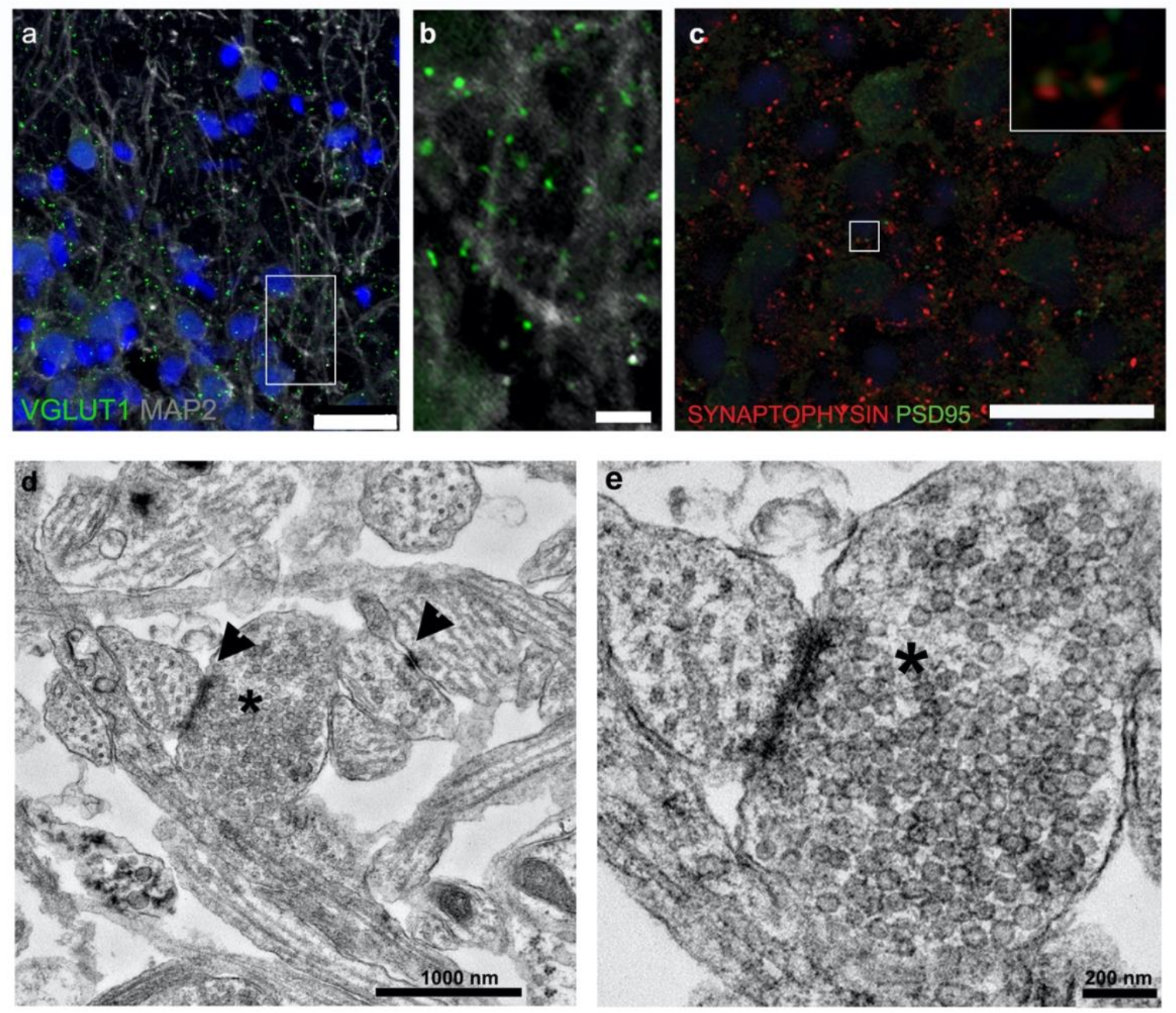

Figure 5. Cortical organoids synaptic maturation. a, b. Representative immunohistochemistry images showing VGLUT1 puncta in MAP2 neuronal dendrites (b, high magnification image of inset in a). c. SYNAPTOPHYSIN pre-synaptic and PSD95 post-synaptic synaptic markers. d, e. Ultrastructure electron microscopy images of cortical organoids showing synaptic clefts (head arrows) and synaptic vesicles (asterisks). Scale bars, $5 \mu \mathrm{m}$ (b), $25 \mu \mathrm{m}(\mathrm{a}, \mathrm{c})$. 
296 We next tested the neurophysiological activity of organoids by multielectrode array (MEA). Five months297 old organoids were cultured in standard conditions until 2 months prior to recordings and then with either 298 cortical organoid differentiation medium (CODM) or a physiological relevant neuronal medium 299 (BrainPhys) up until the MEA recordings. Organoids were placed on the MEA one day before recordings 300 (Figure 6a). Spontaneous firing activity was observed in organoids cultured in BrainPhys (Figure 6b, 301 c), but not in CODM organoids. Similarly, spike raster plots showed firing patterns of organoids across 302 all electrodes with marked network bursts in organoids cultured in BrainPhys only (Figure 6d, e and 303 S5a). The mean firing rate (MFR) of neurons cultured in BrainPhys was significantly greater than CODM 304 grown organoids $(1.7 \pm 1.3 \mathrm{~Hz}$ in BrainPhys and $0.03 \pm 0.04 \mathrm{~Hz}$ in CODM ( $\mathrm{n}=5$ organoids, $\mathrm{N}=3$ 305 independent batches of differentiation, mean \pm SD 60 electrodes, paired two tailed t-test, $p=0.0466$ ) 306 (Figure S5e). Next, we performed pharmacological intervention using tetrodotoxin (TTX) as a synaptic 307 blocker. Organoid network activity was abolished with TTX addition with activity returning to normal 308 levels after washout (Figure $6 f, n=5$ organoids, $N=3$ differentiation batches, mean $\pm S D$, paired two 309 tailed t-test, $\left.{ }^{\star \star * \star} \mathrm{p}<0.0001\right)$.

311 BrainPhys medium has been described as a more physiological environment supporting the basic 312 function of neurons ${ }^{22}$. To gain insights into possible differences between BrainPhys and CODM cultured 313 organoids we compared the proteome of organoids cultured in the two media (Figure $6 \mathbf{g}, \mathbf{h}, \mathrm{n}=3$ 314 organoids for each condition). Proteomic analysis detected 4,334 in common proteins for both conditions 315 with an additional 27 and 242 unique Brain Phys and CODM proteins, respectively. The different culture 316 conditions showed differences in protein enrichment (Figure 6g). Notably, enriched proteins that could 317 potentially explain the improved function of BrainPhys cultured neurons included synaptic vesicle318 associated proteins synaptophysin (encoded by SYP) and synaptotagmin 1 (encoded by SYT1), 319 glutamate receptor delta 1 subunit (encoded by GRID1) involved in synaptogenesis and phosphatase 320 and tensin homolog (encoded by PTEN) responsible for synapse maturation. Furthermore, organoids 321 were transduced with an AAV9.SYN1 (Synapsin 1) promoter driving an mcherry reporter Synapsin 1 322 tethers pools of synaptic vesicles in nerve terminals, binds to the cytoskeleton, and has an activity323 dependent function regulating the availability of synaptic vesicles for neurotransmitter release ${ }^{24}$. When 324 cultured in BrainPhys, organoids showed increased neuronal Synapsin 1 promoter activity when 325 compared to standard CODM organoids (Figure S5a, b). We also confirmed the presence of synapses 326 and synaptic vesicles in BrainPhys organoids using TEM (Figure S5f). 

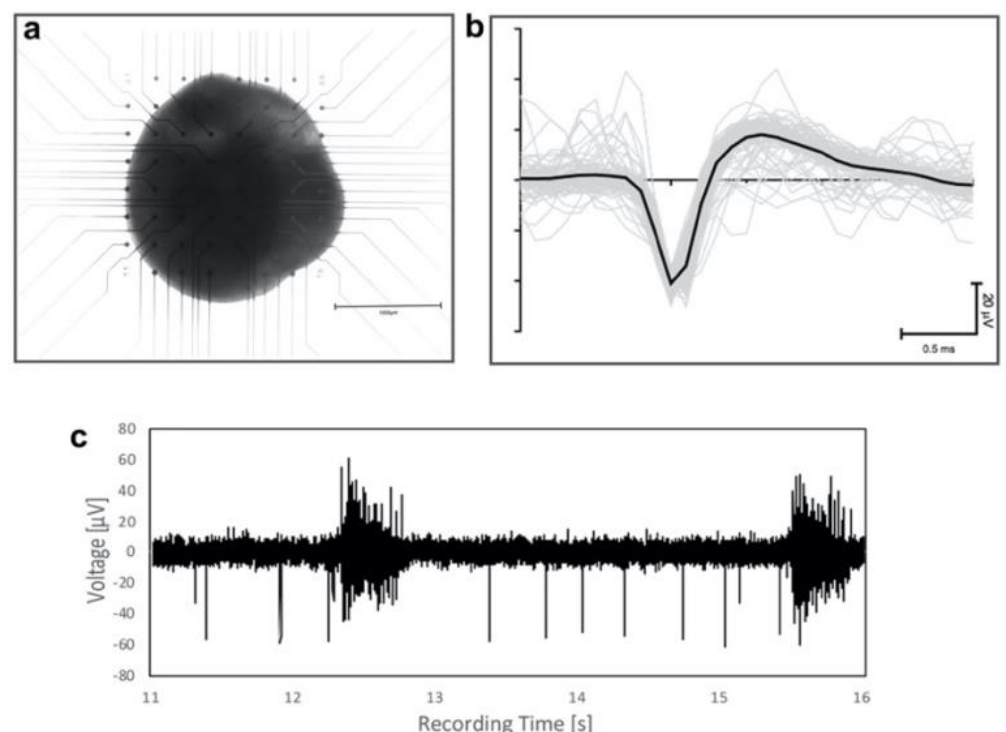

d

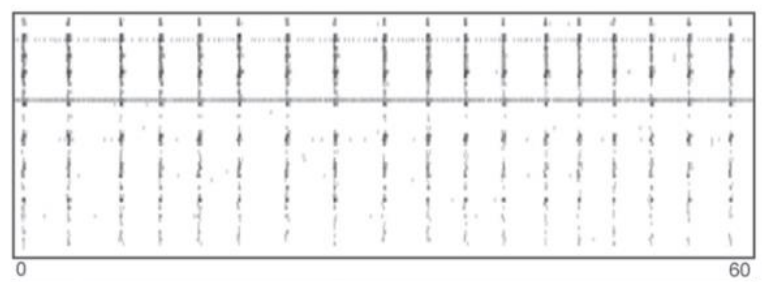

e

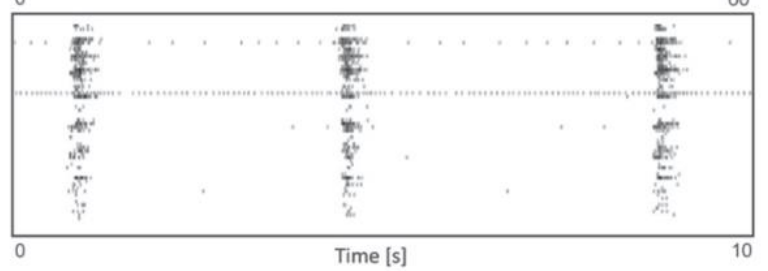

f
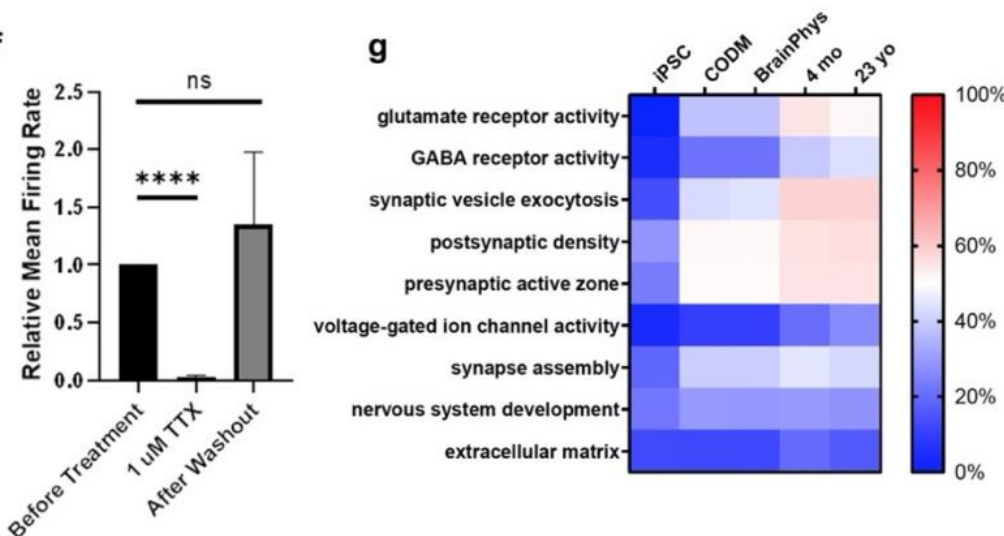

h

- BrainPhys enriched

- CODM enriched

Figure 6. Cortical organoids develop synchronous networks. a. Image showing cortical organoid plated overnight on Multielectrode array (MEA). b, c. Representative spontaneous firing activity of organoid. d, e. Spike raster plots showing firing patterns of organoids across all electrodes with marked network bursts. f. TTX treatment abolished firing activity, which returned following (mean \pm SD, paired two tailed $t$ test, $\left.{ }^{* * * *} \mathrm{p}<0.0001\right)$. g. Graph showing proteins significantly differentially enriched in organoids cultured in CODM or BrainPhys media ( $p<0.05$, adjusted by a false discovery rate of $5 \%$ ). 


\section{3D retinal-cortical organoids form optic nerve-like structures}

336 Having demonstrated the formation of dorsal cortical organoids from 2D/3D confluent differentiation

337 cultures, we next aimed to test whether the 3D suspension culture of complex organoids comprising

338 both retinal and brain organoids promoted the formation of connections between the two organs.

340 In early 2D differentiation cultures, retinal and brain organoids spontaneously developed in proximity enabling the manual isolation of these two organoids for further neural maturation in suspension (Figure 7a). This differentiation potential was tested in 4 PSC lines (see Methods for details), including the H9.mcherry embryonic stem cell (ESC) line where the typical morphology of brain neural rosettes and

344 thick neural epithelium of retinal organoids are easily discerned. At this early time point in 3D culture (5 weeks) the two organoids were attached but failed to show any clear axonal projections (Figure 7b).

346 The connection of the retina to the brain to process the visual information is established by retinal 347 ganglion cells (RGCs), the first-born cell type of the retina, which form neuronal outputs connecting to 348 the brain through the optic nerve. In the complex retinal-brain organoids, at 10 weeks of development, 349 the maturation of each individual organoid was evident. In retinal organoids $\mathrm{NEUN}$ and $\mathrm{HuC} / \mathrm{HuD}$ 350 colocalised with THY1 and MAP2 RGC markers delineating these cells' axonal projections towards the centre of the organoid (Figure S6a, b). Similarly, these two markers were also expressed in neurons within the brain organoids, as demonstrated by the colocalization with TBR1 cortical neural marker (Figure S6c, d). CRX positive photoreceptor cells were present in retinal organoids with RGC THY1 positive axons clearly extending long axonal processes that connect to the TBR1 positive brain organoid (Figure $6 \mathrm{c}, \mathrm{d}, \mathrm{n}=13$ organoids, $\mathrm{N}=9$ differentiation batches). Finally, we used Lightsheet microscopy to image the morphological intricacies of whole $3 D$ retinal-brain complex organoids $(n=3$ organoids, $N=2$ differentiation batches). Cleared organoids enabled the visualisation of MAP2 positive projections connecting both retinal and brain regions forming structures that resemble the optic nerve (Figure 7e,

$\mathrm{h}$ and SMovie 3, $\mathrm{n}=3$ organoids imaged). 

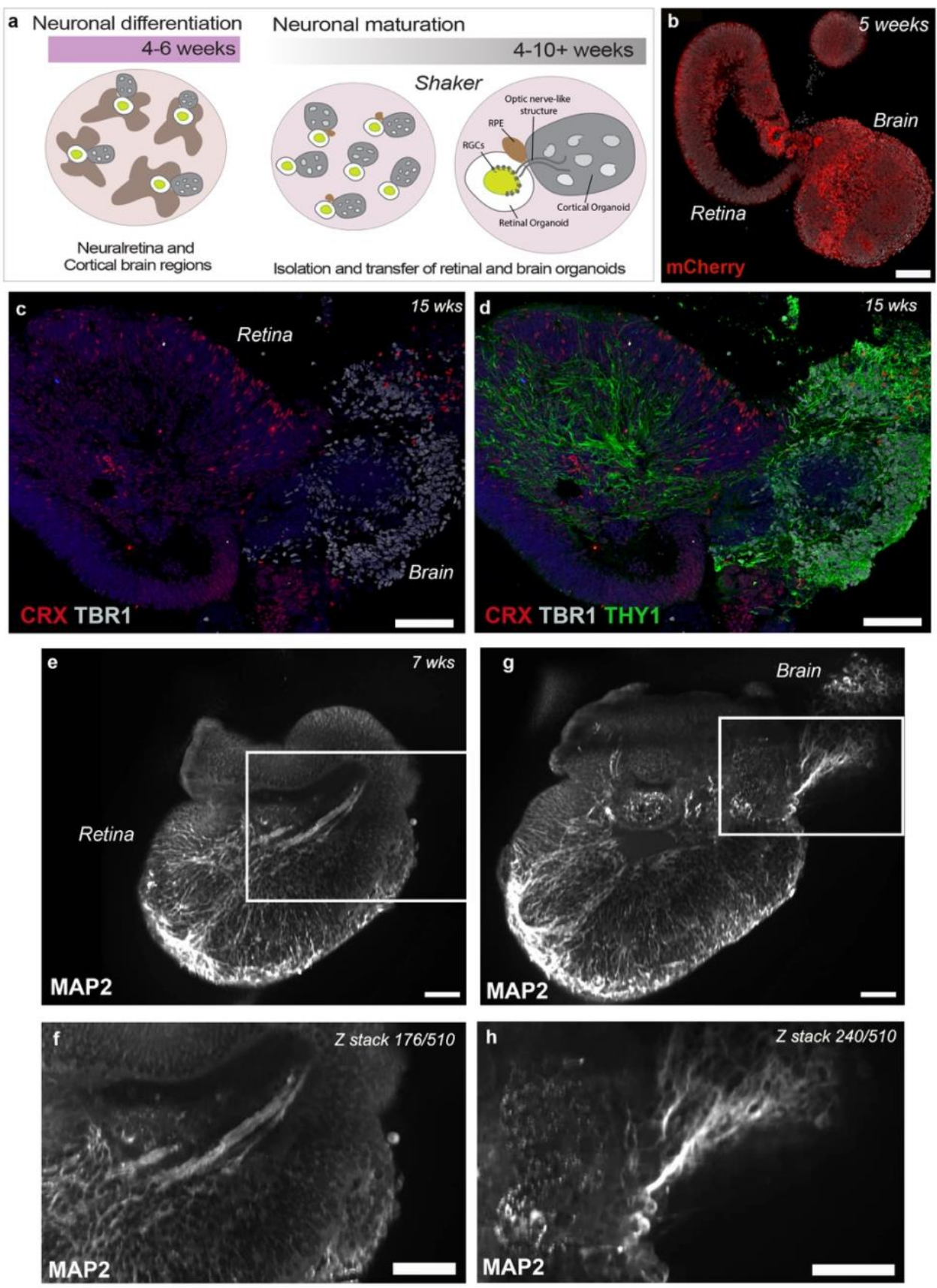

361 Figure 7. Complex retinal-cortical organoids form optic nerve-like structures. a. Schematic

illustrating the timeline of neuronal differentiation and the main cellular components of the retinal-cortical system. b. Week 5 mCherry retinal-brain organoid showing retinal and brain regions growing together. c. Representative immunohistochemistry images of CRX positive photoreceptor cells in retinal organoids and TBR1 neurons in brain organoids. d. THY1 positive retinal ganglion cells send axonal projections to the centre of the retinal organoid that connect with brain organoid THY1 positive neurons in brain regions. e-h. Light sheet image of whole retinal-brain organoid showing consecutive z-stack images of MAP2 retinal ganglion cells axons. e. Forming optic nerve-like structure projecting towards the central part of retinal organoid (e). f. High magnification image optic nerve-like structure (z stack 176 out of 510 stacks) from inset in $\mathbf{g}$. Deeper z-stack 240 showing the same axonal projection connecting to brain region. $\mathbf{h}$. High magnification image of inset in $\mathbf{g}$ showing optic nerve-like structure. Scale bars, $100 \mu \mathrm{m}(\mathrm{b}-\mathrm{d}), 200 \mu \mathrm{m}(\mathrm{e}-\mathrm{h})$. 


\section{Discussion}

The differentiation of PSCs into retinal and brain organoids have been used to model several neurodegenerations. A fundamental requirement for the development of therapies is the establishment of robust pre-clinical models in which they can be tested. Whilst numerous retinal and brain organoid differentiation protocols have been described, these differ considerably in methodology: some methods rely entirely on the addition of growth factors and matrices to guide cell fate decisions and organ formation while others form spontaneous organoids through the endogenous potential of PSCs to form the niches of miniaturized organs in vitro. Variability between protocols and, most importantly, in-batch organoid variability is a common problem of both approaches. Other challenges include the phenotypical and maturity differences between in vitro generated cells and their in vivo counterparts. Furthermore, the generation of the right cell type to enable the robust analysis of functional assays and testing of new therapies requires long-term cultures. Each of these protocols present their own advantages and disadvantages. However, to facilitate scale up and the high throughput use of these brain-like models, their variability and culture costing are usually the most common challenges and, thus, the initial choice of protocol is important.

Here, we adapted a previously-described simple and robust 2D/3D differentiation approach derived from confluent cultures of PSCs ${ }^{19}$ and generated dorsal cortical organoids within the same cultures of retinal vesicles and RPE cells. These cortical organoids are morphologically different and distinguishable from retinal vesicles which display thick neuroephithelia. Instead, brain organoids form the neural rosettes typical of cortical organoids, a feature that enabled their ready isolation. This differentiation method only requires a confluent culture of PSCs followed by change to a pro-neural induction media. Particularly important to reducing variability and costs of long-term cultures, this simple method does not require the addition of Matrigel or exogenous factors. Having tested this protocol in various PSC lines, we confirmed the generation of dorsal cortical organoids that express typical cortical plate markers as well as inhibitory and excitatory neurons and glial populations, with very few PSC lines failing to give rise to neuronal tissues.

The initial extracellular matrix and local cell-cell interactions provided by the 2D confluent environment of differentiating PSCs is sufficient to drive self-formation of discrete 3D structures, such as the eye and the brain. PSC cell lines vary in their efficiency to specify towards the three embryonic germ layers due to cell intrinsic cues specific to each cell line. Self-forming spontaneous methods benefit from the default bias present in most PSC lines to form ectoderm and forebrain ${ }^{25}$. Omics analysis of PSC lines and their initial stage of differentiation have shed light on the variability between cell lines leading to increased 
408

differences between organoids generated among multiple cell lines ${ }^{10,26}$. Our undirected protocol enables reproducibility of directed organoids but with the benefits of spontaneous differentiations.

Despite tremendous progress in the field, the lack of disease-relevant functional assays in organoids hinders their ability to test for new treatment efficacy. However, disease molecular signatures and biomarkers can be determined using integrative analysis of omics as well as computational or bioinformatic methodologies. In this study, cortical organoids generated by 2D/3D confluent method were extensively characterized using scRNA-seq and proteomics analysis, setting a baseline for future studies using disease organoids. In agreement with a previous correlation study comparing scRNA-seq datasets of brain organoids and foetal human brain ${ }^{12}$, our organoids showed a high correlation with brain organoids derived by other methodologies, particularly with dorsally patterned brain organoids generated in the directed differentiation approach demonstrated in Velasco et al., 2019 ${ }^{16}$, known for their reproducibility.

The proteome of brain organoids has been seldom investigated. Previous proteomic analysis of earlystaged (day 45) brain organoids generated using a whole-brain spontaneous 3D aggregation method highlighted the protein-protein interactions involved in early neuronal specification, including the migration of progenitor cells, radial glia, and oligodendrocyte progenitors ${ }^{27}$. Our proteomic analysis compared iPSCs and five months old cortical organoids. The analysis supported the increase in expression of proteins related to neuronal development and synaptic function in the cortical organoids and a relative decrease in cell cycle related proteins. The detection of synaptic protein components was further corroborated by IHC for synaptic markers and ultrastructure electron microscopy.

Neuronal network formation in brain organoids have previously been studied elegantly in late-stage organoids cultured on the MEA for a few months ${ }^{10,15}$ and in bioengineered neuronal organoids ${ }^{28}$. Our own electrophysiology analysis verified the functionality of cortical organoids, demonstrating their synchronous neuronal network activity when cultured for the final two months of differentiation in an electrophysiologically-relevant medium, BrainPhys ${ }^{22}$. A separate proteomics analysis identified proteins enriched in the BrainPhys-cultured organoids that might explain how BrainPhys media promotes favourable protein expression and electrophysiological activity. Overall, these results demonstrate the generation of functional cortical organoids that can be reliably differentiated for disease modelling and precision medicine studies.

Neurodevelopmental disorders leading to higher order cognitive abilities mostly affect the cortex and thus modelling of these conditions using cortical organoids is crucial ${ }^{29}$. In the eye, numerous retinal 
443 diseases have been investigated using retinal organoids ${ }^{30,31-38}$. However, modelling of more complex 444 disease traits requires the differentiation of specific brain regions and their functional integration as well 445 as their connection with other organs. One example of complex organotypic formation was 446 demonstrated by the fusion of cortical, spinal cord organoids and skeletal muscle spheroids which generated a functional cortico-motor assembloid system leading to robust muscle contraction ${ }^{39}$.

In this study, we demonstrated the spontaneous formation of retinal-and-brain complex organoids in the 2D/3D confluent cultures. When in 3D suspension, these retinal-brain organoids maintained their proximity and were connected through an optic nerve-like structure mimicking the neuronal projections that connect the eye and brain. Our confluent method of differentiation enabled the precise isolation of these two organoids, overcoming variability within 3D directed and whole brain protocols that sporadically generate eye structures ${ }^{8,10}$ and the forced fusion of different organoids to form assembloids. The latter have been successfully used to model in vivo neuronal interactions between different brain regions ${ }^{1,39-42}$. The formation of optic nerve structures in vitro in complex organoid structures will better elucidate the dynamics of retinogenesis and neurogenesis that can then be used to model a number of optic neuropathies, such as glaucoma. Importantly, these retinal-brain organoids also promise to improve organoid development, such as the long-term survival and maturation of retinal ganglion cells in the retinal organoids as these cells would no longer lack their contact with the output in the cortex. Neurodegeneration in glaucoma is complex and extends beyond the eye into the lateral geniculate nucleus and visual cortex ${ }^{18}$ of the brain. Similarly, late onset neurodegenerative diseases, such Alzheimer's show early phenotypes in the eye ${ }^{17}$, which enables pre-symptomatic diagnosis ${ }^{43}$. Accurate and more complex models of such diseases will elucidate novel aspects of disease pathogenesis and aid the challenges of developing effective treatments.

Our data demonstrate the simple and undirected derivation of both cortical and retinal organoids from a confluent culture of PSCs. The simplicity of this differentiation method coupled with the ease of precisely locating dorsal cortical organoids, due to their proximity to retinal vesicles, addresses the current problems of in-batch organoid-to-organoid variability. The comprehensive characterisation shown here, including novel proteome data of brain organoids, expands the available repertoire of relevant models of human disease. Importantly, this method also enabled the formation of complex organoid-forming structures that resemble the important connection, between the retina and the brain, the optic nerve. 
Materials and Methods

\section{Derivation of UCLOOi017-A-1 induced pluripotent stem cell (iPSC) line}

481 Peripheral blood mononuclear cells (PBMCs) were isolated from the whole blood of a healthy donor using density gradient centrifugation. Briefly, $25 \mathrm{ml}$ of whole blood diluted $1: 1$ with phosphate buffered saline (PBS) was layered on top of $15 \mathrm{ml}$ of Ficoll-Paque Premium (GE) and centrifuged with brake and accelerator off at $500 \mathrm{~g}$ for 30 minutes and the cloudy interphase containing PBMCs was collected. After washing once with PBS, the cells were counted and 2 million were cultured for 6 days in hematopoietic expansion media -Stemspan H3000(Stemcell Technologies), with the addition of EPO (R\&D), IL-3 (Life Technologies), Dexamethasone (Sigma), Ascorbic Acid (Sigma), SCF (Miltenyi) and IGF-1 (Miltenyi). Following expansion, 200000 cells were nucleofected using Amaxa 4D nucleofector with Addgene http://n2t.net/addgene:27077; RRID:Addgene_27077) [pCXLE-hUL, pCXLE-hSK and pCXLEhOCT3/4-shp53-F were gifts from Shinya Yamanaka]. The nucleofected cells were plated on a well of a 6 well plate coated with Geltrex matrix (Life Technologies) and transitioned to Essential 8 media (Life Technologies) ${ }^{1}$. iPS cells were visible within a week and small colonies were visible within 2 weeks. Four to five weeks later, iPSC colonies were large enough to be excised, broken up and transferred using a $10 \mu l$ pipette to individual wells of a 12 well plate (Corning). Once colonies had enlarged in the 12 well plate, each well (containing an iPSC clone) was dissociated and further cultured separately on 6 well plates (Corning) for 10 passages prior to characterisation.

\section{Human PSC maintenance}

502 The human pluripotent stem cells including iPSC (UCLOOi017-A-1, ECCACs HPSI0214i-kucg_2 and HPSI0314i-hoik_1 and TiPSC-52) and ESC (WiCell H9 WA09, H7 WA07) lines were maintained on feeder free conditions on Essential 8 media (E8, Life technologies) and Geltrex (Life technologies) coated 6 well plates. Briefly, when 70\% confluent, PSCs were dissociated using Versene solution (Life technologies) at $37^{\circ} \mathrm{C}$ for 5 to 10 minutes. The dissociated clumps were washed once in PBS with centrifugation at $900 \mathrm{rpm}$ for 5 minutes. The resulting cell pellet was broken up and resuspended in $1 \mathrm{ml}$ E8 media by 1000ul pipette and distributed on a Geltrex coated 6 well plate with E8 and $10 \mu \mathrm{M} \mathrm{ROCK}$ inhibitor (Y-27632 dihydrochloride, Tocris) for 24 hours. Daily feeding with E8 was continued for further maintenance culture.

511 
513 Human iPSCs were maintained until 90-95\% confluent as described above. Media was replaced with 514 Essential 6 media (E6, Life Technologies) for 2 consecutive days. At day 3 of differentiation, E6 media 515 was replaced with a pro-neural induction media (PIM, composed of Advanced DMEM/F12, N2 516 supplement, L-Glutamine, non-essential amino acids and Antibiotic-antimycotic). At around week 3-4 517 of culture, three-dimensional (3D) organoids containing rosettes were observed throughout the plate 518 and in close proximity to neuroretinal vesicles. The 3D cortical organoids were manually excised with $51919 \mathrm{G}$ needles and kept together in $60 \mathrm{~mm}$ well plates in retinal differentiation media (RDM, composed of 520 DMEM, F12 Nutrient mix, B27-vitamin A and antibiotic-antimycotic) and placed on an orbital shaker at 52185 RPM. At 6 weeks of differentiation, retinal differentiation medium was supplemented with $10 \%$ FBS, $522100 \mu \mathrm{M}$ Taurine (Sigma, T4871) and 2 mM Glutamax. At 10 weeks, cortical organoids were cultured in 523 a cerebral organoid differentiation medium (CODM) as described in Lancaster \& Knoblich, Nature 524 Protocols, 2015 (Composed of Neurobasal medium, DMEM/F12, N2 supplement, insulin, Glutamax, MEM-NEAA, B-mercaptoethanol, B27 supplement) or BrainPhys ${ }^{\mathrm{TM}}$ hPSC Neuron Kit (Stemcell Technologies, composed of BrainPhys Neuronal medium, NeuroCult SM1 neuronal supplement, N2 supplement, human recombinant brain-derived neurotrophic factor, human recombinant glial cell linederived neurotrophic factor, ascorbic acid and dibutyryl-cAMP). Differentiation cultures were fed every Monday, Wednesday and Friday. All representative images in the paper were from UCLOOi017-A-1 iPSC line unless otherwise stated.

\section{Immunohistochemistry}

533 hPSC-derived Cortical organoids were used for assessments of the time course of differentiation, as 534 described in the main manuscript. Cortical Brain Organoids were washed with PBS, fixed for 40 minutes 535 to 60 minutes depending on the size of the organoid in $4 \%$ paraformaldehyde and washed again with 536 PBS prior to overnight suspension in $20 \%$ sucrose. The organoids, once sunken in sucrose, were 537 embedded in OCT and snap frozen in nitrogen.

538 Cortical brain organoids were cryo-sectioned at 14 um thickness, collected on SuperFrost slides 539 (Thermo) and preserved at minus $20^{\circ} \mathrm{C}$. Cryosections were washed with PBS and blocked in $5 \%$ serum 540 (goat or donkey) in blocking solution (1\% Bovine serum albumin in PBS with $0.1 \%$ Triton-X) for 2 hours. 541 Primary antibody (Supplemental Table 1) diluted in blocking solution was incubated overnight at $4^{\circ} \mathrm{C}$. 542 Sections were washed with PBS three times and incubated with secondary antibody (Alexa fluor 488, 543 546, 633 secondary antibodies, Invitrogen-Molecular Probes) diluted in blocking solution (1:500) at room 544 temperature. Sections were then washed with PBS and counter stained with DAPI (Sigma-Aldrich).

545 For immunohistochemistry of whole cortical organoids in 3D view using Lightsheet, a clearing protocol 546 was performed. Briefly, cortical brain organoids were fixed in $4 \%$ paraformaldehyde, suspended in $20 \%$ 547 sucrose and stored in $4^{\circ} \mathrm{C}$ as described above. Samples were incubated in clearing reagent 1 (Urea, 
$548 \mathrm{~N}, \mathrm{~N}, \mathrm{~N}^{\prime}, \mathrm{N}^{\prime}$-tetrakis, Triton $\mathrm{X}, \mathrm{NaCl}, \mathrm{dH}_{2} \mathrm{O}$ ) overnight followed by block in blocking solution (1\% BSA in 549 PBS, $5 \%$ serum and $0.3 \%$ Triton X) for 2 hours or overnight. Primary antibodies and DAPI were added 550 in the blocking solution without serum overnight to increase penetration of antibody. Samples were 551 washed with PBS and secondary antibodies diluted in blocking solution at 1:500 were added to the 552 organoids overnight. The following day, samples were washed with PBS and clearing reagent 2 553 (Sucrose, Urea, Triethanolamine, Triton $\mathrm{X}, \mathrm{dH}_{2} \mathrm{O}$ ) was added overnight. Samples were embedded in 554 low melting point agarose and left hanging in clearing reagent 2 for approximately 1 or 2 days until the samples were clear.

\section{Image acquisition}

Images were acquired by confocal microscopy (LSM 880 Airyscan or SP5 Leica). A series of XY optical sections, approximately $1.0 \mu \mathrm{m}$ apart, throughout the depth of the section were taken and built into a stack to give a projection image. For Zeiss and Leica, ZEN blue and LAS AF image software were used, respectively. For Lightsheet fluorescence microscopy, images were acquired using Zeiss Lightsheet Z.1 using similar parameters to normal confocal using Zen Black software. Further image processing was performed in Imaris (version 9, Bitplane AG, Switzerland) software. Briefly, after images were imported, surfaces for each of the channels were created using the Surface Creation Wizard. Smoothing and threshold limits were applied to eliminate background light form the image. Optical sections of the organoid were created using the Oblique Slicer tool.

\section{Dissociation of Brain organoids}

569 To produce 2D neuron cultures, cortical organoids were washed with PBS once and incubated in trypsin 570 or Accutase for 5 minutes at $37^{\circ} \mathrm{C}$. Organoids were then dissociated into single cells by gently pipetting 571 up and down. Cells were then centrifuged at $300 \mathrm{~g}$ for 5 minutes and cell pellets were resuspended in $572250 \mu \mathrm{l}$ of media and seeded onto Geltrex coated 8 well chamber slides. Media was changed every other 573 day until dissociated cells reached 11 weeks in culture.

574 For scRNA-seq, cortical organoids were dissociated with Neurosphere dissociation kit (Miltenyi Biotec). 575 Cortical organoids were transferred to Eppendorf tubes then washed with $1 \mathrm{ml}$ of PBS. Enzyme mix was 576 prepared as per manufacture instructions and $500 \mathrm{ul}$ added to each tube and incubated at $37^{\circ} \mathrm{C}$ for 10 577 minutes and flicked every 2 minutes. Organoids were then pipetted up and down with p1000 pipette to 578 mechanically dissociate the big clumps of cells. The tubes were returned to $37^{\circ} \mathrm{C}$ for another 5 minutes and further dissociation. Following this incubation, a p200 pipette was used to break up the clumps further and $500 \mu \mathrm{l}$ of HBSS was added to each tube and the contents were filtered using MACS $30 \mathrm{um}$ 
temperature and resuspended in $200 \mu$ of CODM media with the tubes kept on ice when transferred for cell counting.

\section{Quantification}

Chamber slides were fixed by incubating in 4\% PFA for 10 minutes and stained with TUJ1 and GFAP antibodies using the immunohistochemistry protocol described above. Next, cells were imaged using the Zeiss Axio Imager Alpha confocal microscope. Cell clumps cells were located using epifluorescence illumination and TUJ1 and GFAP positive cells were imaged from 10 different regions in the chamber slide and quantified using FiJi software. To accurately measure the percentage of TUJ1 neurons and GFAP glial cells, the number of DAPI, TUJ1 and GFAP cells were counted using cell counter plug-in. Then the percentages of TUJ1 and GFAP cells were calculated by in relation to the total number of DAPI cells.

\section{Single Cell RNA-Sequencing}

596 Organoids were dissociated as described above and a subset of cell suspension was stained with $0.4 \%$ 597 Trypan Blue (Gibco) and assessed for viability and concentration using Countess II Automated Cell 598 Counter (Invitrogen). Single cell suspensions were passed through $40 \mu \mathrm{m}$ cell strainer (Corning) and concentration was adjusted to 1000 cells/ $\mu$ l. The suspension was loaded in single-cell-B Chip (10X Genomics) for target output of 10,000 cells per sample. Single-cell droplet capture was performed on the Chromium Controller (10X Genomics). cDNA library preparation was performed in accordance with the Single-Cell 3' v3 protocol. Libraries were evaluated for fragment size and concentration using Agilent HSD5000 ScreenTape System. Samples were sequenced on an Illumina NovaSeq6000 instrument according to manufacturer's instructions (Illumina). Sequencing was carried out using 2x150 paired-end (PE) configuration with a sequencing depth of 40,000 reads per cell. The sequences were processed by GENEWIZ. The data supporting the findings in this publication have been deposited in NCBI's Gene Expression Omnibus and are accessible through GEO Series accession number GSE174232

\section{Single Cell RNA Sequencing analysis}

611 Raw files were processed with Cell Ranger 2.0.1 software (10X Genomics). The Cell Ranger count module was used to align sequence reads from scRNA-seq experiment to the GRCh38 human those that are expressed in at least three cells and those that have at least 500 genes detected. Cells 
617 (according to 10X Genomics guidelines). To compare our cortical organoid scRNA-seq data with other 618 published datasets, a combined cell-by-gene count matrix of the processed scRNA-seq datasets with 619 cell annotation was obtained from OrganoidAtlas (https://cells-test.gi.ucsc.edu/?ds=organoidatlas) ${ }^{4}$. We 620 subset six scRNA-seq datasets from six different brain organoid studies each using a different 621 culture/differentiation protocol. These include Giandomenico et al., 2019 (denoted as "Gian 3m U”), 622 Birey et al., $2017^{5}$ (Birey 3m D), Velasco et al., 20196 (Vela 3m D), Quadrato et al., $2017^{7}$ (Quad 3m U), 623 Trujillo et al., $2019^{8}$ (Truj 6m D), and Velasco et al., $2019^{6}$ (Vela 6m D). A fetal brain dataset is also 624 included for comparison (Zhong et al., 2018) ${ }^{9}$ (Fetal). The cell type compositions of each public dataset 625 were visualized as staked bar plots. Cell annotation of the cortical organoid scRNA-seq generated in 626 this study was carried out by using scClassify (Version 1.0.0) ${ }^{10}$. Briefly, we trained prediction models 627 using each published dataset as reference separately and joint trained. Then, we predicted the cell types 628 in our cortical organoid scRNA-seq based on the individual and the joint prediction models. A t629 distributed stochastic neighbour embedding (t-SNE) was applied to visualize the cells with labels 630 generated from the joint prediction model. To compare agreement in cell-type composition between our 631 cortical organoid and the public brain organoid and fetal brain datasets, intraclass correlation coefficient 632 (ICC) was calculated by generating a table of cell-type composition across published organoids and our 633 organoid, using the ICC function in the irr R package (Version 0.84.1). To evaluate the reproducibility of 634 cell type prediction, logarithm transformed data were used for assessing the expression of a panel of 635 marker genes listed in ${ }^{4}$ in each cell type based on the scClassify prediction. The marker genes include 636 neuronal growth cone markers (STMN2, GAP43, DCX), early neurogenesis markers (VIM, HES1, 637 SOX2), excitatory neuron markers (TBR1, SLC17A7), inhibitory neuron markers (GAD1, SLC32A1), 638 radial glial marker (EOMES), cell cycle markers (TOP2A, MKI67), oligodendrocyte precursor cells 639 markers (OLIG1, OLIG2), astrocyte markers (S100B, SLC1A3, GFAP), cilium markers (MNS1, NPHP1), 640 BMP signal markers (BMP4, MSX1), mesoderm marker (MYH3), proteoglycan markers (BGN, DCN) and unfolded

protein

response

cell

marker

(DDIT3).

\section{Electron microscopy (EM)}

644 Brain organoids were fixed overnight in Karnovsky's fixative (2.5\% glutaraldehyde and $2.4 \%$ 645 formaldehyde in MOPS buffer), then washed in MOPs buffer. They were post fixed with $2 \%$ osmium 646 tetroxide, incubated in $2 \%$ aqueous uranyl acetate, dehydrated in an ethanol series, and embedded in 647 TAAB Low Viscosity Resin (TAAB Laboratories). Sections were cut at 90nm using a UC6 ultramicrotome 648 (Leica Microsystems), then stained with 2\% uranyl acetate in 50\% ethanol (10 min) and Reynold's lead citrate (4 min). Grids were examined with a JEOL JEM-1400 transmission electron microscope operating at $80 \mathrm{kV}$, with images recorded using a Matataki Flash sCMOS camera. 


\section{Recording of organoid activity on Multiple Electrode Array (MEA)}

653 After media replacement as described previously, a single organoid for each recording was placed on to 654 a 60-electrode MEA200/30iR-Ti multiple electrode array (MEA) plate (MultiChannel Systems), ensuring 655 coverage of the electrodes. Medium was removed and $20 \mu \mathrm{l}$ of Matrigel extracellular matrix (Corning) 656 was dropped onto the organoid. The MEA plate was subsequently incubated at $37{ }^{\circ} \mathrm{C}$ for 15 mins to 657 allow the Matrigel to set the organoid in place. $500 \mu \mathrm{l}$ of medium was added dropwise and the plate was 658 placed back in the incubator at $\left[37^{\circ} \mathrm{C}, 5 \% \mathrm{CO} 2,70 \%\right.$ humidity] for 24 hours before recording electrical 659 activity.

660 Organoid electrical activity was measured using an MEA2100-lite system with TC01 temperature control 661 (MultiChannel Systems), heated to $37^{\circ} \mathrm{C}$. Recordings were made for 5-10 minutes at a frequency of 10 $662 \mathrm{kHz}$ using MultiChannel Experimenter software (MultiChannel Systems). Data was processed using the 663 MultiChannel Analyzer software (MultiChannel Systems). Raw electrode recordings were filtered 664 sequentially with a $200 \mathrm{~Hz}$ second order Butterworth high pass filter then a $3 \mathrm{kHz}$ second order 665 Butterworth low pass filter. Spikes were picked using a threshold of 5 standard deviations below mean 666 voltage for each electrode.

667 Voltage-gated sodium channel activity was blocked using Tetrodotoxin (TTX). Briefly, baseline activity 668 was recorded by MEA as described above. Immediately afterwards, Brainphys media was removed, 669 and washed once with PBS, then replaced with Brainphys media containing $1 \mu \mathrm{M}$ TTX. Following a 10 670 minute incubation in culture conditions, a further MEA recording was taken. Subsequently, the media 671 containing TTX was removed, and the organoid was washed 3 times with PBS, with fresh Brainphys 672 media added after the final wash. Following 2 hours in the incubator, a final recording was taken.

673 Results were produced by counting the spikes using a -5SD threshold after filtering, deriving an average 674 spike number for the whole MEA, deriving an average frequency for the whole MEA (by dividing the 675 spike number by recording time), and normalising each organoid's pre-, during and post-treatment 676 frequencies to the frequency of the organoid during the pre-treatment recording. Normalised values 677 were assessed for significance independently using a paired t-test, assuming similar distributions 678 between the two datasets.

\section{Preparation of organoids and analysis by mass spectrometry}

Six organoids were isolated and cultured as previously described until week 10 after initiation of differentiation. Three of these organoids were either cultured in CODM or BrainPhys medium for two weeks. Medium was removed from organoids which were then homogenised and lysed with a drill and pestle in $200 \mu \mathrm{l}$ 2\% SDS Lysis Buffer [2\% (w/v) sodium dodecyl sulfate (SDS), $50 \mathrm{mM} \mathrm{HEPES} \mathrm{pH} \mathrm{7.4,}$

$6852 \mathrm{mM}$ ethylene glycol-bis ( $\beta$-aminoethyl ether)-N,N,N',N'-tetraacetic acid (EGTA), 2 mM 686 ethylenediaminetetraacetic acid (EDTA), $2 \mathrm{mM}$ phenylmethylsulfonyl fluoride (PMSF), EDTA-free 
687 Protease Inhibitor (Roche) and PhosSTOP (Roche)] and freezing on dry ice. Frozen organoid lysate 688 was thawed and reduction was performed with $10 \mathrm{mM}$ tris(2-carboxyethyl)phosphine (TCEP) at $85^{\circ} \mathrm{C}$ 689 for $10 \mathrm{~min}$ with shaking. Samples were alkylated with $20 \mathrm{mM}$ iodoacetamide for $30 \mathrm{~min}$ at $23^{\circ} \mathrm{C}$ in the 690 dark. Protein was precipitated from using the chloroform-methanol method ${ }^{11}$. Protein pellet was 691 reconstituted in $20 \mu \mathrm{L}$ of $7.8 \mathrm{M}$ Urea, $50 \mathrm{mM}$ HEPES $\mathrm{pH}$ 8.0. Protein was digested by the addition of 3 $692 \mu \mathrm{g}$ Lys-C (FUJIFILM Wako Pure Chemical Corporation) and incubation for 8 hours at $25^{\circ} \mathrm{C}, 900 \mathrm{rpm}$. 693 Sample was then diluted 8-fold with $50 \mathrm{mM}$ HEPES pH 8.0 and digested by the addition of $5 \mu \mathrm{g}$ 694 TrypZean recombinant trypsin (Sigma-Aldrich) and incubation for 8 hours at $30^{\circ} \mathrm{C}$, with shaking. The 695 trypsin digestion was repeated for each sample. Protein content of each digest was estimated by

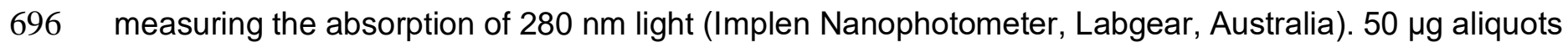
697 of each sample were desalted on an in-house made STAGE tip with C18 material. Samples were eluted 698 in $50 \%$ acetonitrile, dried and then reconstituted in a solution of $90 \%$ acetonitrile, $0.1 \%$ trifluoroacetic 699 acid (TFA) for hydrophilic interaction chromatography (HILIC) fractionation.

700 HILIC fractionation was performed on a Dionex Ultimate 3000 HPLC system with a $250 \mathrm{~mm}$ long and 1 $701 \mathrm{~mm}$ inside diameter TSKgel Amide-80 column (Tosoh Biosciences). HILIC gradient was between a 702 solution of $90 \%$ acetonitrile, $0.1 \%$ TFA (Buffer A) and a solution of $0.1 \%$ TFA (Buffer B). The sample 703 was injected into a $250 \mu \mathrm{l}$ sample loop to be loaded onto the column at a flow rate of $60 \mu \mathrm{l} / \mathrm{min}$ in Buffer 704 A for 10 min. Gradient was from 100\% Buffer A to 60\% Buffer A (balanced with Buffer B) for 35 min at 705 a flow rate of $50 \mu \mathrm{l} / \mathrm{min}$. Fractions were collected into a 96-well plate using a Probot (LC Packings) at 1 706 minute intervals, monitored by absorbance of UV at $214 \mathrm{~nm}$. UV signal was used to combine select 707 fractions into similar amounts of peptide. Fractions were dried and reconstituted in $0.1 \%$ formic acid for 708 LC-MS/MS analysis.

709 The LC-MS/MS was performed using a Dionex UltiMate 3000 RSLC nano system and Q Exactive Plus 710 hybrid quadrupole-orbitrap mass spectrometer (Thermo Fisher Scientific). The content of HILIC fractions 711 was loaded directly onto an in-house $300 \times 0.075 \mathrm{~mm}$ column packed with ReproSil Pur C18 AQ 1.9 $712 \mu \mathrm{m}$ resin (Dr Maisch, Germany). The column was heated to $50{ }^{\circ} \mathrm{C}$ using a column oven (PRSO-V1, 713 Sonation lab solutions, Germany) integrated with the nano flex ion source with an electrospray operating 714 at $2.3 \mathrm{kV}$. The $\mathrm{S}$ lens radio frequency level was 60 and capillary temperature was $250{ }^{\circ} \mathrm{C}$. The $5 \mu \mathrm{L}$ 715 sample was injected into a $20 \mu \mathrm{l}$ loop and loaded onto the column in $99 \%$ reversed phase buffer $\mathrm{A}$ 716 (solution of $0.1 \%$ formic acid) and $1 \%$ buffer B (solution of $0.1 \%$ formic acid, $90 \%$ acetonitrile) for $25 \mathrm{~min}$ 717 at $300 \mu \mathrm{L} / \mathrm{min}$. The sample was loaded in $99 \%$ buffer $\mathrm{A}$ for $25 \mathrm{~min}$. The gradient was from then from $71899 \%$ buffer $A$ to $95 \%$ buffer A in $1 \mathrm{~min}$, to $75 \%$ buffer A in $75 \mathrm{~min}$, to $35 \%$ buffer $\mathrm{A}$ in $8 \mathrm{~min}$, to $1 \%$ buffer $719 \mathrm{~A}$ in $1 \mathrm{~min}$, held at $1 \%$ buffer $\mathrm{A}$ for $2 \mathrm{~min}$, to $99 \%$ buffer $\mathrm{A}$ in $1 \mathrm{~min}$ and held for $10 \mathrm{~min}$. MS acquisition 720 was performed for the entire $120 \mathrm{~min}$. 
721 All samples and fractions were analysed using data-dependent acquisition LC-MS/MS. For data722 dependent acquisition, the MS scans were at a resolution of 70,000 with an automatic gain control target 723 of $1,000,000$ for a maximum ion time of $100 \mathrm{~ms}$ from $\mathrm{m} / \mathrm{z} 375$ to 1500 . The MS/MS scans were at a 724 resolution of 35,000 with an automatic gain control target of 200,000 and maximum ion time of $115 \mathrm{~ms}$.

725 The loop count was 12 , the isolation window was $1.2 \mathrm{~m} / \mathrm{z}$, the first mass was fixed at $\mathrm{m} / \mathrm{z} 140$ and the 726 normalized collision energy was 28 . Singly charged ions and those with charge $>8$ were excluded from 727 MS/MS and dynamic exclusion was for $35 \mathrm{~s}$.

728 The raw LC-MS/MS data was processed with MaxQuant v1.6.7.02 ${ }^{12}$ using the following settings: 729 variable modifications were oxidation (M), acetyl (protein N-terminus), deamidation (NQ); 730 carbamidomethyl (C) was a fixed modification; digestion was set to specific for trypsin with a maximum 731 of 3 missed cleavages; Label Free Quantification (LFQ) was enabled; Homo sapiens reference 732 proteome with canonical and isoform sequences downloaded March 92020 was used as well as the 733 inbuilt contaminants file; minimum peptide length was 7 and maximum peptide mass was 5000 Da; 734 second peptides search was enabled while dependent peptides searches were disabled; peptide 735 spectrum matching and protein false discovery rates were set at 1\%; minimum score for modified 736 peptides was 40; all modified peptides and counterpart non-modified peptides were excluded from 737 protein quantification; matching between runs was disabled. All other parameters were default.

738 Entries in proteinGroups results file were filtered: CON_ and REV_entries were removed; entries with $739<2$ unique + razor peptides were removed; entries with no Intensity values were removed; entries for

740 which no gene name could be derived were removed. LFQ Intensity values for remaining entries was

741 used as input for an R script that used limma statistics to compare protein relative intensity

742 statistically.

743 The code for this script is reported here:

744 https://github.com/ChildrensMedicalResearchInstitute/MS intensity diffexpress.

745 The mass spectrometry proteomics data have been deposited to the ProteomeXchange Consortium 746 via the PRIDE ${ }^{13}$ partner repository with the dataset identifier PXD025933 and 10.6019/PXD025933.

\section{Gene ontology enrichment analysis of iPSC and cortical organoid proteomes}

749 The list of proteins detected by mass spectrometry were filtered to remove proteins that were not 750 detected in all three biological replicates. The number of "razor plus unique" peptides was required to be two or greater using an average value across the three biological replicates. The filtered protein list was converted to a gene list. Duplicate genes arising from multiple protein accession and isoforms were 
pathway terms were determined by comparing to the human coding genome. The probability of significant enrichment was adjusted using a false discovery rate of $5 \%$.

\section{AAV transduction of brain organoids}

AAV viral vectors (1-3.6x10E11 vg/organoid) were added to a total volume of $375 \mu \mathrm{l}$ using fresh CODM media used to culture the cortical and whole brain organoids. The organoids were then transferred to low binding 24 well plates (Costar, Corning) and media was completely replaced with CODM containing the AAV vectors. Cortical and whole brain organoids were incubated at $37^{\circ} \mathrm{C}$ for half a day before adding another $625 \mu \mathrm{l}$ of fresh media. After overnight culture at $37{ }^{\circ} \mathrm{C}$, the organoids and CODM/vector Wednesday and Friday.

\section{Statistical analysis}

All means are presented as mean \pm SD (standard deviation) unless otherwise stated; $N$, number of independent experiments i.e differentiation batches, proteome samples or MEA measurements; $n$, number of images or retinal organoids examined, where appropriate. Statistical differences between two groups were tested by two tailed paired and unpaired $t$ tests. The preformed test is specified in figure legends and main text. Statistical significance was assessed using Graphpad Prism software. Figures were generated in Adobe Photoshop.

\section{Methods References}

777 1. Okita, K., Ichisaka, T. \& Yamanaka, S. Generation of germline-competent induced pluripotent

Mcience 53, 2007-2019 (2012). Transcriptomes from Multiple Brain Organoids and Fetal Brain. Cell Rep 30, 1682-1689.e3 (2020). 59 (2017). 
789 6. Velasco, S. et al. Individual brain organoids reproducibly form cell diversity of the human $790 \quad$ cerebral cortex. Nature 570, 523-527 (2019).

791 7. Quadrato, G. et al. Cell diversity and network dynamics in photosensitive human brain $792 \quad$ organoids. Nature 545, 48-53 (2017).

793 8. Trujillo, C. A. et al. Complex Oscillatory Waves Emerging from Cortical Organoids Model Early

9. Zhong, S. et al. A single-cell RNA-seq survey of the developmental landscape of the human

10. Lin, Y. et al. scClassify: sample size estimation and multiscale classification of cells using single and multiple reference. Mol Syst Biol 16, e9389 (2020).

11. Rappsilber, J., Ishihama, Y. \& Mann, M. Stop and go extraction tips for matrix-assisted laser desorption/ionization, nanoelectrospray, and LC/MS sample pretreatment in proteomics. Anal Chem 75, 663-670 (2003).

12. Tyanova, S., Temu, T. \& Cox, J. The MaxQuant computational platform for mass spectrometrybased shotgun proteomics. Nat Protoc 11, 2301-2319 (2016).

13. Perez-Riverol $Y$, Csordas A, Bai J, et., al. The PRIDE database and related tools and resources in 2019: improving support for quantification data. Nucleic Acids Res 47(D1):D442D450 (2019).

\section{Acknowledgments}

809 We thank Dr. Scott Page and Joshua Studdert from the Children's Medical Research Institute Advanced 810 Microscopy Centre and the ACRF Telomere Analysis Centre supported by the Australian Cancer 811 Research Foundation, for providing access to microscopy equipment. We thank Emma Kettle for 812 Electron Microscopy, performed at the Westmead Scientific Platforms, which are supported by the 813 Westmead Research Hub, the Cancer Institute New South Wales, the National Health and Medical 814 Research Council and the lan Potter Foundation. We thank Joshua Studdert and Hillary Knowles from 815 the Single Cell Analytics Facility which is supported by NSW Luminesce Alliance. We acknowledge the 816 support of the Children's Medical Research Institute Biomedical Proteomics Facility. Cell line iPS TiPSC8175 was kindly provided by Prof Yvan Arsenijevic and Prof David Gamm. This work is supported by 818 Luminesce Alliance (PPM1 K5116/RD274) - Innovation for Children's Health for its contribution and 819 support. Luminesce Alliance - Innovation for Children's Health, is a not for profit cooperative joint venture 820 between the Sydney Children's Hospitals Network, the Children's Medical Research Institute, and the 821 Children's Cancer Institute. It has been established with the support of the NSW Government to 822 coordinate and integrate paediatric research. Luminesce Alliance is also affiliated with the University of 823 Sydney and the University of New South Wales Sydney. 


\section{Author Contributions}

825 M.F. and S.L contributed to conception, design, execution and analysis of all experiments; J.R.W. 826 contributed to proteomics and multielectrode array experiments; D.X and H.J.K contributed to 827 transcriptome data analysis; G.C.S. contribute to tissue culture experiments and immunohistochemistry; 828 R.R.A. provided UCLOOi017-A-1 iPS cell line and contributed to manuscript revision; P.Y. contributed to design and transcriptome data analysis and manuscript writing; M.E.G. contributed to conception, design, execution of proteomics and multi electrode array experiments and manuscript writing; A.G.C contributed to the conception, design, execution and analysis of all experiments, manuscript writing and funding.

\section{Competing interests}

834 No competing interests declared

\section{References}

1. $\quad$ Birey, F. et al. Assembly of functionally integrated human forebrain spheroids. Nature 545, 5459 (2017).

2. Qian, X. et al. Brain-Region-Specific Organoids Using Mini-bioreactors for Modeling ZIKV

Exposure. Cell 165, 1238-1254 (2016).

3. Gomes, A. R. et al. Modeling Rett Syndrome With Human Patient-Specific Forebrain Organoids. Front Cell Dev Biol 8, 610427 (2020).

4. Ying, Q.-L., Stavridis, M., Griffiths, D., Li, M. \& Smith, A. Conversion of embryonic stem cells into neuroectodermal precursors in adherent monoculture. Nature Biotechnology 21, 183-186 (2003).

5. Chambers, S. M. et al. Highly efficient neural conversion of human ES and iPS cells by dual inhibition of SMAD signaling. Nat Biotechnol 27, 275-280 (2009).

6. Paşca, A. M. et al. Functional cortical neurons and astrocytes from human pluripotent stem cells in 3D culture. Nat Methods 12, 671-678 (2015).

7. Sinn, R. \& Wittbrodt, J. An eye on eye development. Mechanisms of Development 130, 347358 (2013).

8. Lancaster, M. A. et al. Cerebral organoids model human brain development and microcephaly. Nature 501, 373-379 (2013).

9. Lancaster, M. A. \& Knoblich, J. A. Generation of cerebral organoids from human pluripotent stem cells. Nat Protoc 9, 2329-2340 (2014).

10. Quadrato, G. et al. Cell diversity and network dynamics in photosensitive human brain organoids. Nature 545, 48-53 (2017).

11. Qian, X., Song, H. \& Ming, G.-L. Brain organoids: advances, applications and challenges. Development 146, (2019).

12. Tanaka, Y., Cakir, B., Xiang, Y., Sullivan, G. J. \& Park, I.-H. Synthetic Analyses of Single-Cell Transcriptomes from Multiple Brain Organoids and Fetal Brain. Cell Rep 30, 1682-1689.e3 (2020).

13. Giandomenico, S. L. et al. Cerebral organoids at the air-liquid interface generate diverse nerve tracts with functional output. Nat. Neurosci. 1-17 (2019).

14. Zhong, S. et al. A single-cell RNA-seq survey of the developmental landscape of the human prefrontal cortex. Nature 555, 524-528 (2018). 
868 15. Trujillo, C. A. et al. Complex Oscillatory Waves Emerging from Cortical Organoids Model Early Human Brain Network Development. Stem Cell 1-20 (2019).

16. Velasco, S. et al. Individual brain organoids reproducibly form cell diversity of the human cerebral cortex. Nature 570, 523-527 (2019).

17. Ning, A., Cui, J., To, E., Ashe, K. H. \& Matsubara, J. Amyloid-beta deposits lead to retinal degeneration in a mouse model of Alzheimer disease. Investigative Ophthalmology \& Visual Science 49, 5136-5143 (2008).

18. Gupta, N., Ang, L.-C., Noël de Tilly, L., Bidaisee, L. \& Yücel, Y. H. Human glaucoma and neural degeneration in intracranial optic nerve, lateral geniculate nucleus, and visual cortex. $\mathrm{Br} J$ Ophthalmol 90, 674-678 (2006).

19. Reichman, S. et al. From confluent human iPS cells to self-forming neural retina and retinal pigmented epithelium. Proceedings of the National Academy of Sciences of the United States of America 111, 8518-8523 (2014).

20. Reichman, S. et al. Generation of Storable Retinal Organoids and Retinal Pigmented Epithelium from Adherent Human iPS Cells in Xeno-Free and Feeder-Free Conditions. Stem Cells 35, 1176-1188 (2017).

21. Gonzalez Cordero, A. et al. Recapitulation of Human Retinal Development from Human Pluripotent Stem Cells Generates Transplantable Populations of Cone Photoreceptors. Stem Cell Reports 9, 820-837 (2017).

22. Bardy, C. et al. Neuronal medium that supports basic synaptic functions and activity of human neurons in vitro. PNAS 112, E2725-34 (2015).

23. Lin, Y. et al. scClassify: sample size estimation and multiscale classification of cells using single and multiple reference. Mol Syst Biol 16, e9389 (2020).

24. Cesca, F., Baldelli, P., Valtorta, F. \& Benfenati, F. The synapsins: Key actors of synapse function and plasticity. Progress in Neurobiology 91, 313-348 (2010).

25. Espuny-Camacho, I. et al. Pyramidal neurons derived from human pluripotent stem cells integrate efficiently into mouse brain circuits in vivo. Neuron 77, 440-456 (2013).

26. Bock, C. et al. Reference Maps of human ES and iPS cell variation enable high-throughput characterization of pluripotent cell lines. Cell 144, 439-452 (2011).

27. Nascimento, J. M. et al. Human Cerebral Organoids and Fetal Brain Tissue Share Proteomic Similarities. Front Cell Dev Biol 7, 303 (2019).

28. Zafeiriou, M.-P. et al. Developmental GABA polarity switch and neuronal plasticity in Bioengineered Neuronal Organoids. Nat Commun 11, 3791-12 (2020).

29. Zhao, X. \& Bhattacharyya, A. Human Models Are Needed for Studying Human Neurodevelopmental Disorders. Am J Hum Genet 103, 829-857 (2018).

30. Kruczek, K. et al. Gene Therapy of Dominant CRX-Leber Congenital Amaurosis using Patient Stem Cell-Derived Retinal Organoids. Stem Cell Reports 16, 252-263 (2021).

31. Kallman, A. et al. Investigating cone photoreceptor development using patient-derived NRL null retinal organoids. Commun Bio/ 3, 82-13 (2020).

32. Lane, A. et al. Modeling and Rescue of RP2 Retinitis Pigmentosa Using iPSC-Derived Retinal Organoids. Stem Cell Reports 15, 67-79 (2020).

33. Lukovic, D. et al. Retinal Organoids derived from hiPSCs of an AIPL1-LCA Patient Maintain Cytoarchitecture despite Reduced levels of Mutant AIPL1. Sci Rep 10, 5426-13 (2020).

34. Guo, Y. et al. Modeling Retinitis Pigmentosa: Retinal Organoids Generated From the iPSCs of a Patient With the USH2A Mutation Show Early Developmental Abnormalities. Front Cell Neurosci 13, 361 (2019).

35. Quinn, P. M. et al. Human iPSC-Derived Retinas Recapitulate the Fetal CRB1 CRB2 Complex Formation and Demonstrate that Photoreceptors and Müller Glia Are Targets of AAV5. Stem Cell Reports 12, 906-919 (2019).

36. Deng, W.-L. et al. Gene Correction Reverses Ciliopathy and Photoreceptor Loss in iPSCDerived Retinal Organoids from Retinitis Pigmentosa Patients. Stem Cell Reports 10, 12671281 (2018). 
37. Megaw, R. et al. Gelsolin dysfunction causes photoreceptor loss in induced pluripotent cell and animal retinitis pigmentosa models. Nat Commun 8, 271 (2017).

38. Parfitt, D. A. et al. Identification and Correction of Mechanisms Underlying Inherited Blindness in Human iPSC-Derived Optic Cups. Cell stem cell 18, 769-781 (2016).

39. Andersen, J. et al. Generation of Functional Human 3D Cortico-Motor Assembloids. Cell 183, 1913-1929.e26 (2020).

40. Xiang, Y. et al. hESC-Derived Thalamic Organoids Form Reciprocal Projections When Fused with Cortical Organoids. Stem Cell 24, 487-497.e7 (2019).

41. Bagley, J. A., Reumann, D., Bian, S., Lévi-Strauss, J. \& Knoblich, J. A. Fused cerebral organoids model interactions between brain regions. Nat Methods 14, 743-751 (2017).

42. Miura, Y. et al. Generation of human striatal organoids and cortico-striatal assembloids from human pluripotent stem cells. Nat Biotechno/ 38, 1421-1430 (2020).

43. Hadoux, X. et al. Non-invasive in vivo hyperspectral imaging of the retina for potential biomarker use in Alzheimer's disease. Nat Commun 10, 4227-12 (2019).

\section{Supplementary information}

\section{Supplementary Figures}

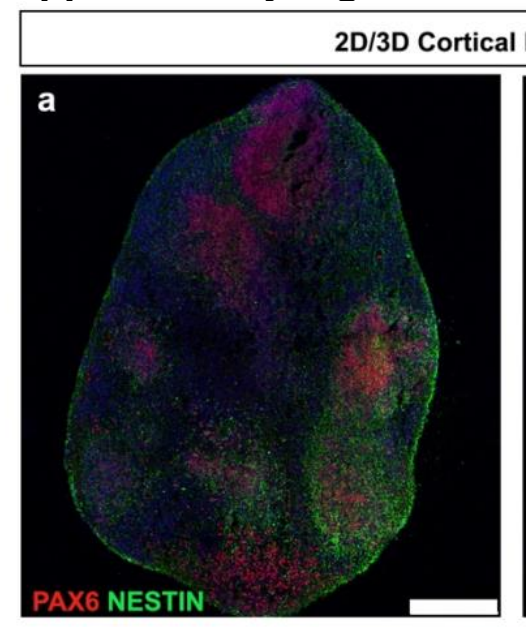

\section{Brain organoid}

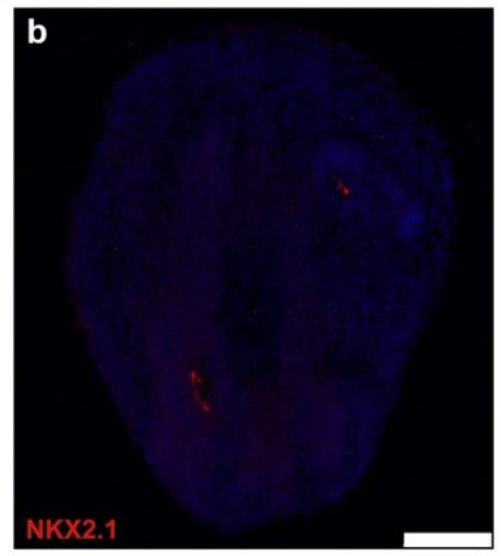

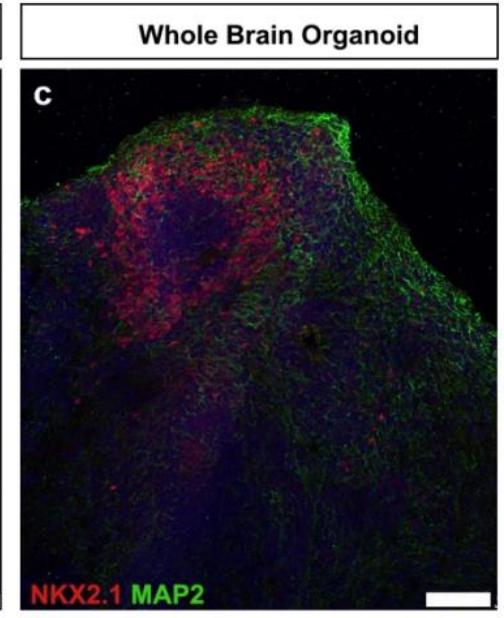
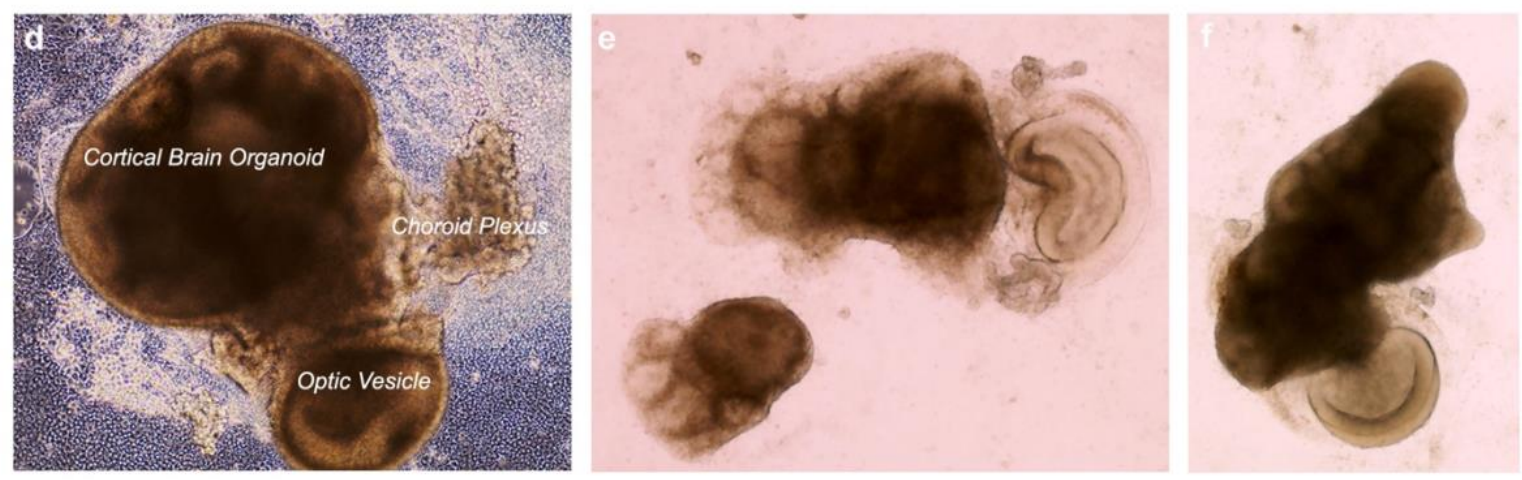

Supplementary Figure 1. Confluent iPS differentiation protocol gives rise to cortical and retinal organoids. a. Immunohistochemistry image showing PAX6 and NESTIN positive cortical organoid. b. Image showing that cortical organoid negative for ventral cortex marker, NKX2.1. c. Image showing NKX2.1 and MAP2 positive whole brain organoid. d-f. Bright field imaged showing examples of cortical brain organoids growing in proximity to optic vesicles. Scale bars, $75 \mu \mathrm{m}$ (c), $250 \mu \mathrm{m}(\mathrm{a}, \mathrm{b})$. 
bioRxiv preprint doi: https://doi.org/10.1101/2021.05.16.444356; this version posted May 17, 2021. The copyright holder for this preprint (which was not certified by peer review) is the author/funder, who has granted bioRxiv a license to display the preprint in perpetuity. It is made available under aCC-BY 4.0 International license.

947

948

949

950

951

952

953

954

955

956

957

958

959 


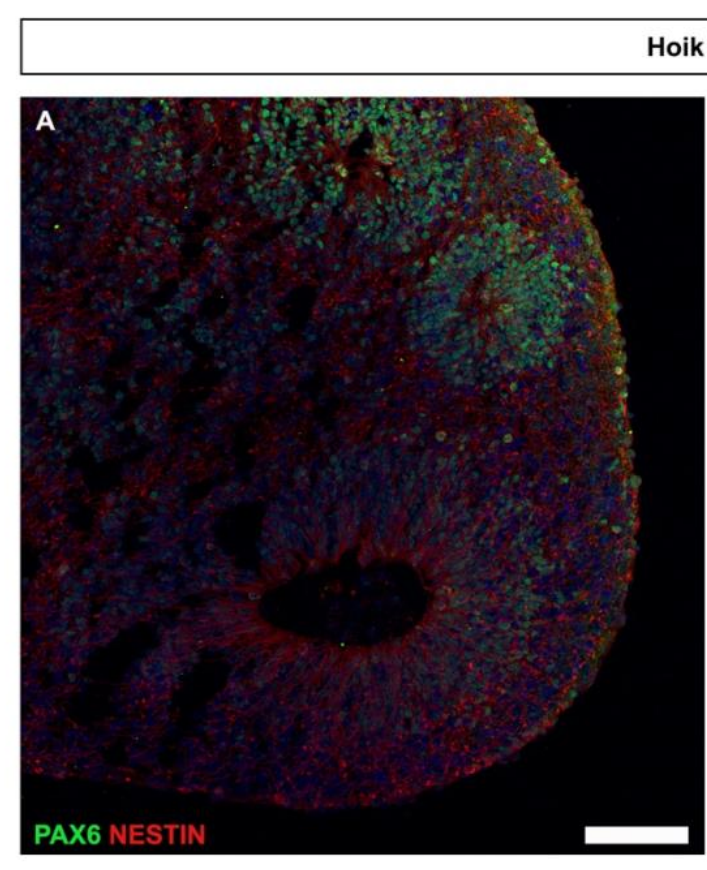

Hoik iPS cell line
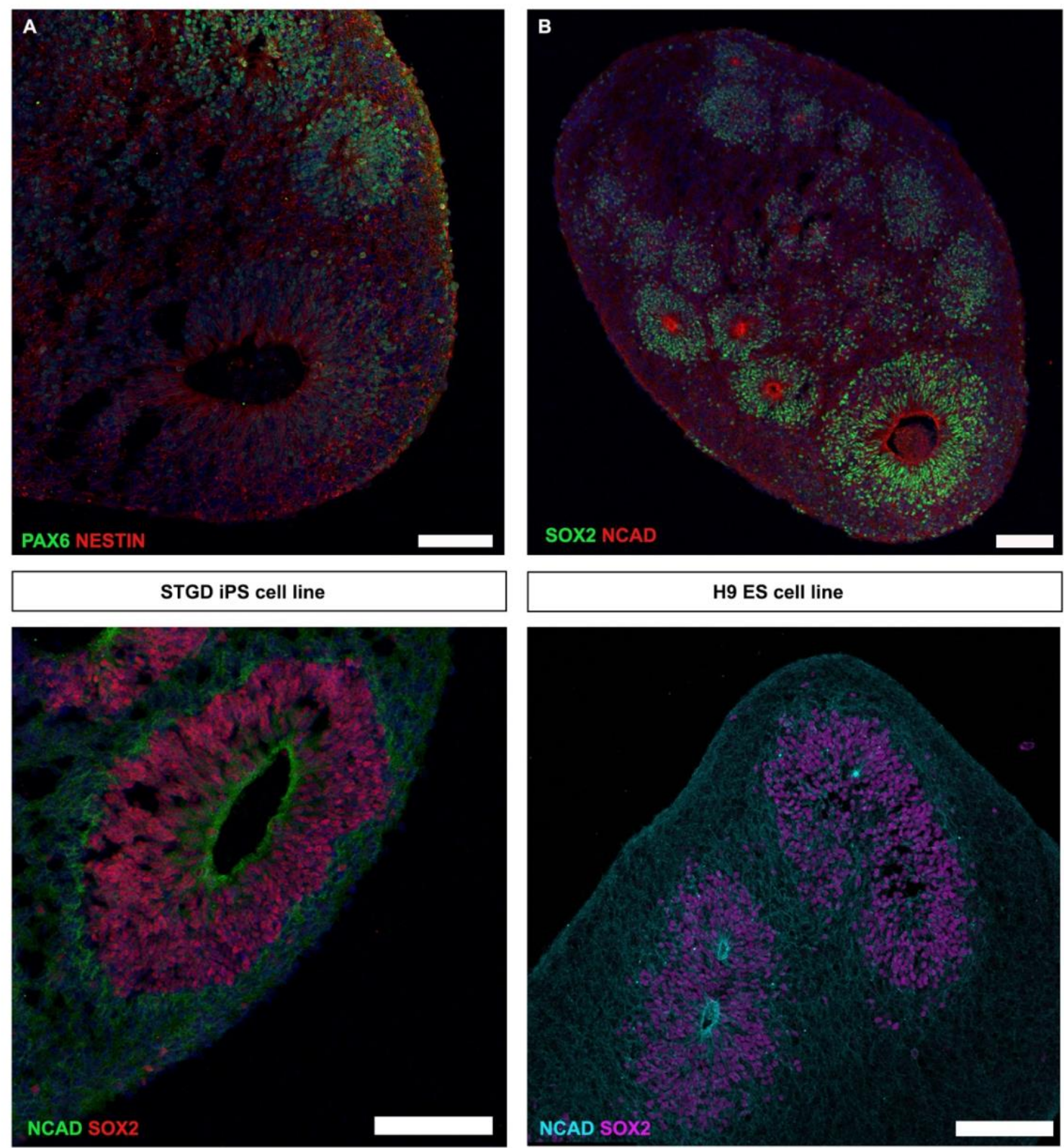

960

961 Supplementary Figure 2. Early cortical organoids derived from various iPSC lines. a-d. 962 Representative images of early neural markers of cortical organoids generated from iPSC 963 lines and ES cell line H9. Scale bars, $100 \mu \mathrm{m}(\mathrm{a}-\mathrm{d})$. 
bioRxiv preprint doi: https://doi.org/10.1101/2021.05.16.444356; this version posted May 17, 2021. The copyright holder for this preprint (which was not certified by peer review) is the author/funder, who has granted bioRxiv a license to display the preprint in perpetuity. It is made available under aCC-BY 4.0 International license.

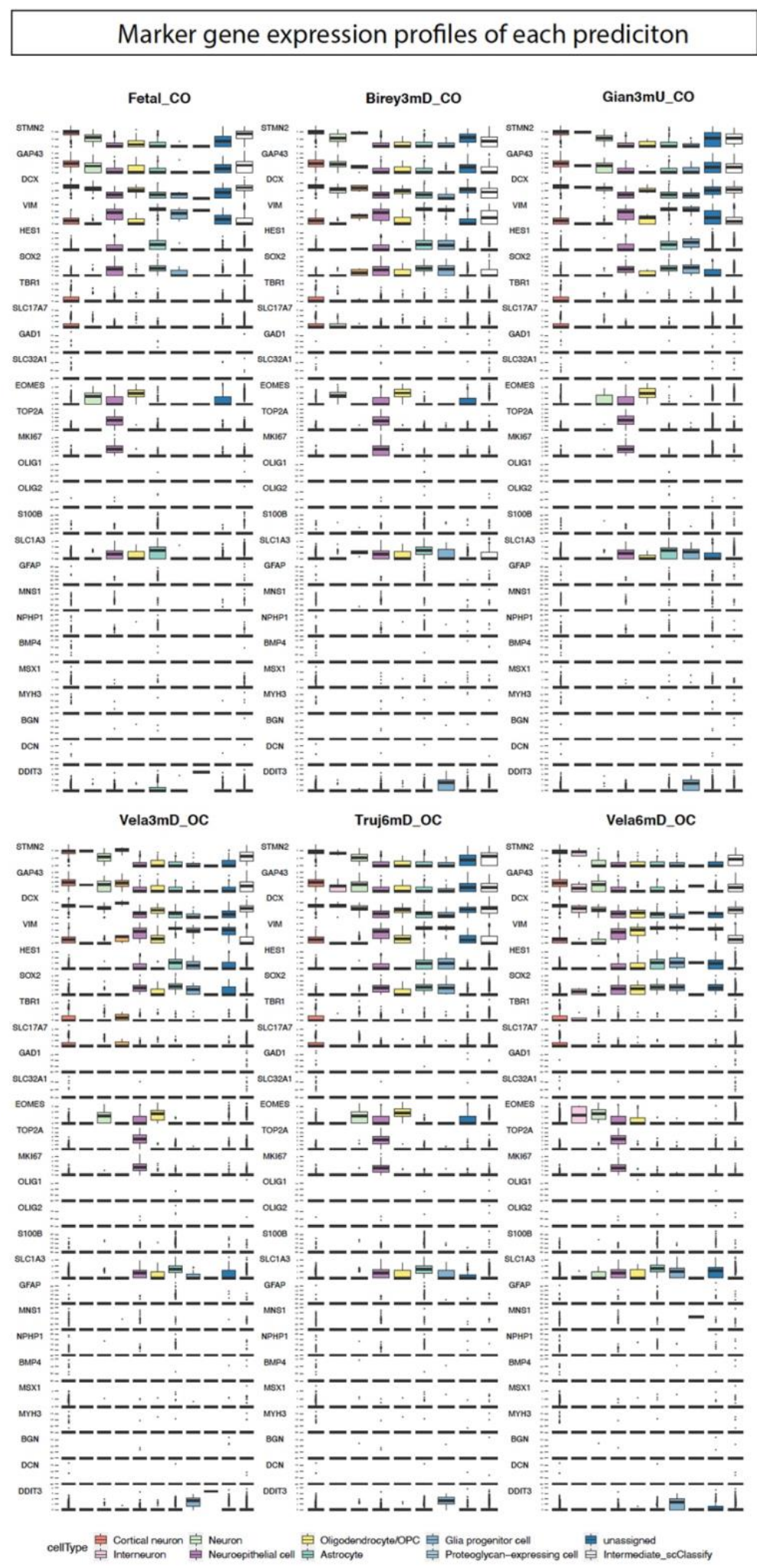



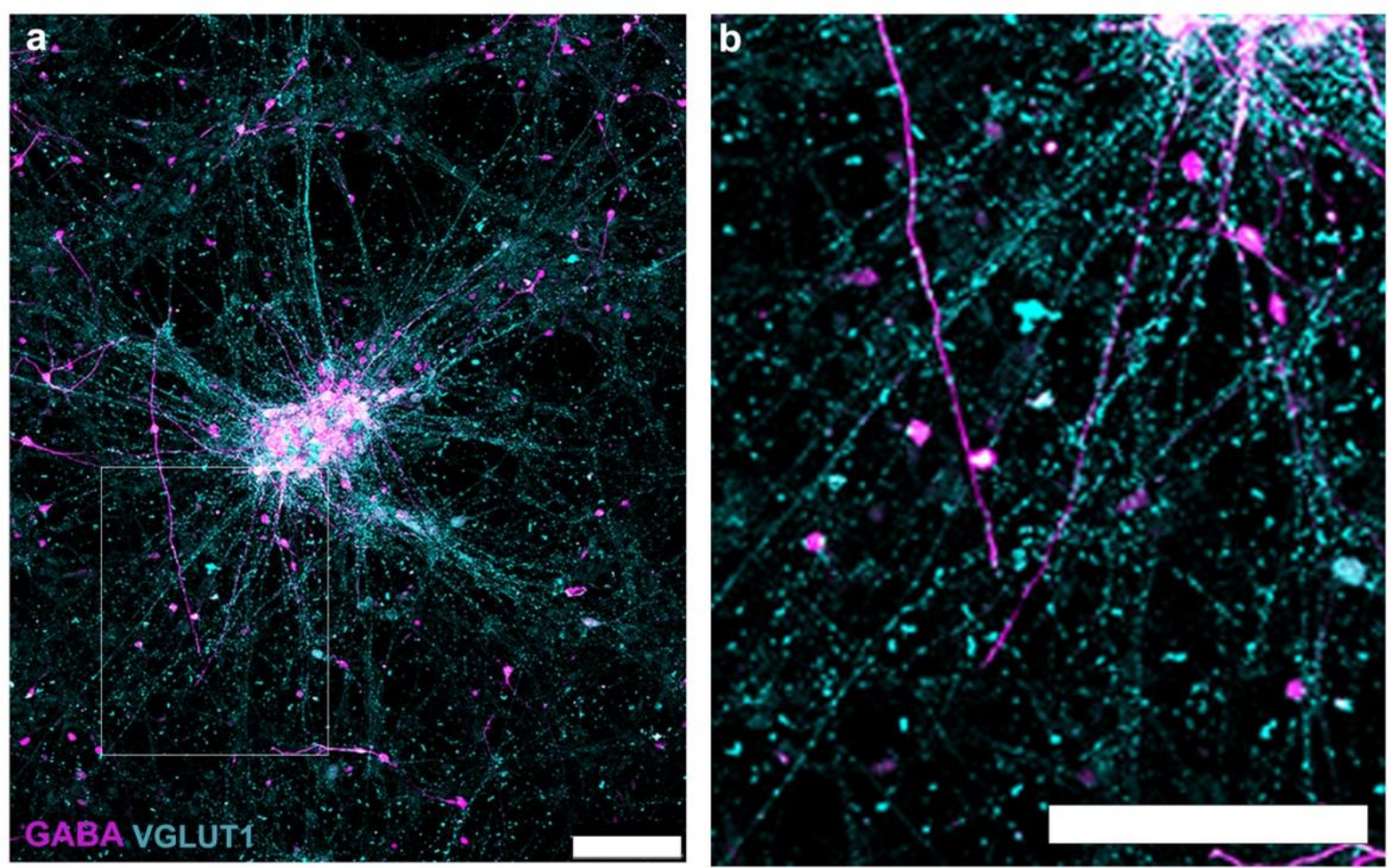

968

969

Supplementary Figure 4. 2D cultures of cortical neurons dissociated from brain

970 organoids. a, b. Immunohistochemistry image showing GABA inhibitory neurons and

971 VGLUT1 inhibitory neurons. $b$ is the high magnification image of inset in a. Scale bars, 100 $\mu \mathrm{m}(\mathrm{a}, \mathrm{b})$. 

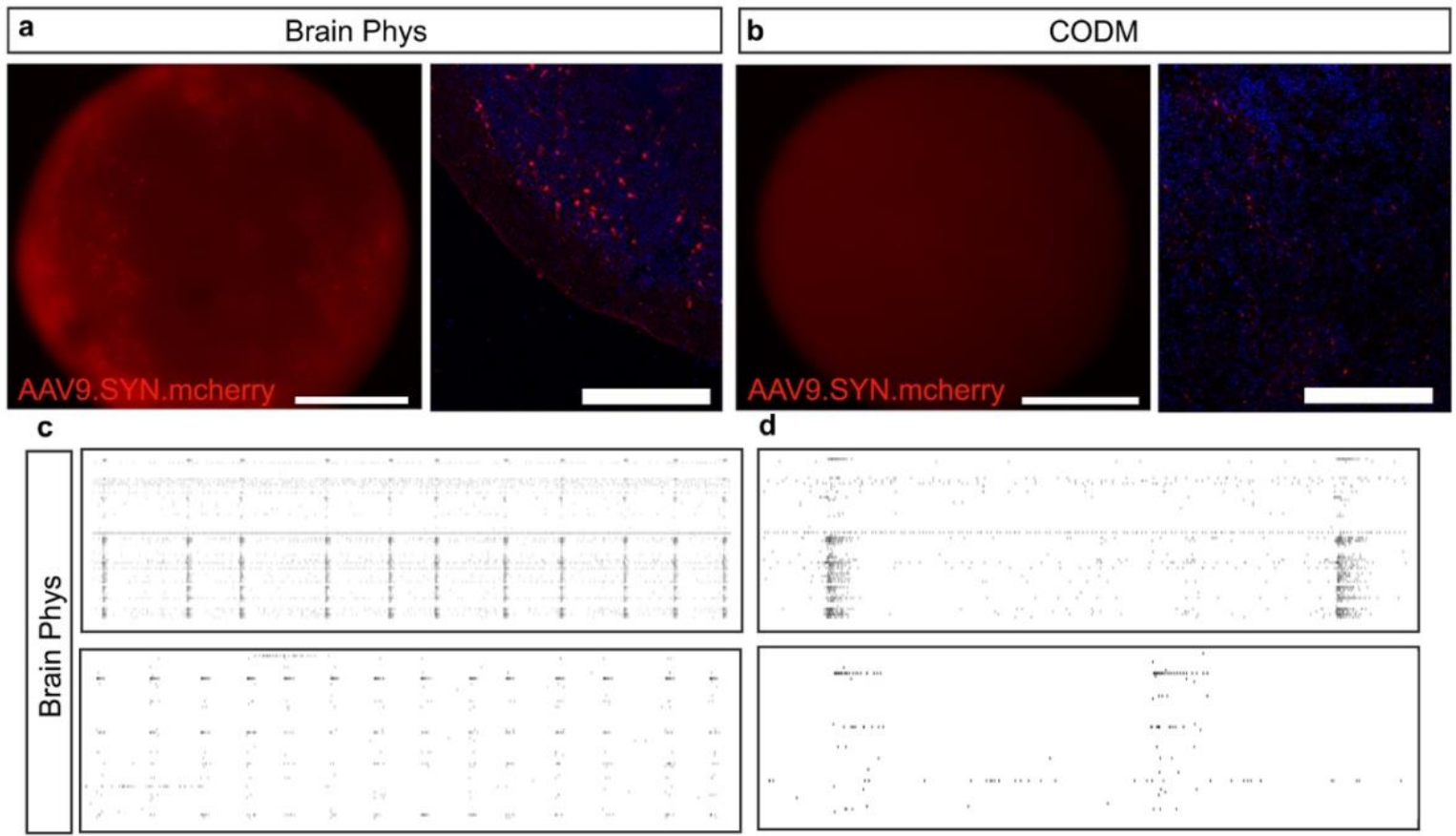

d
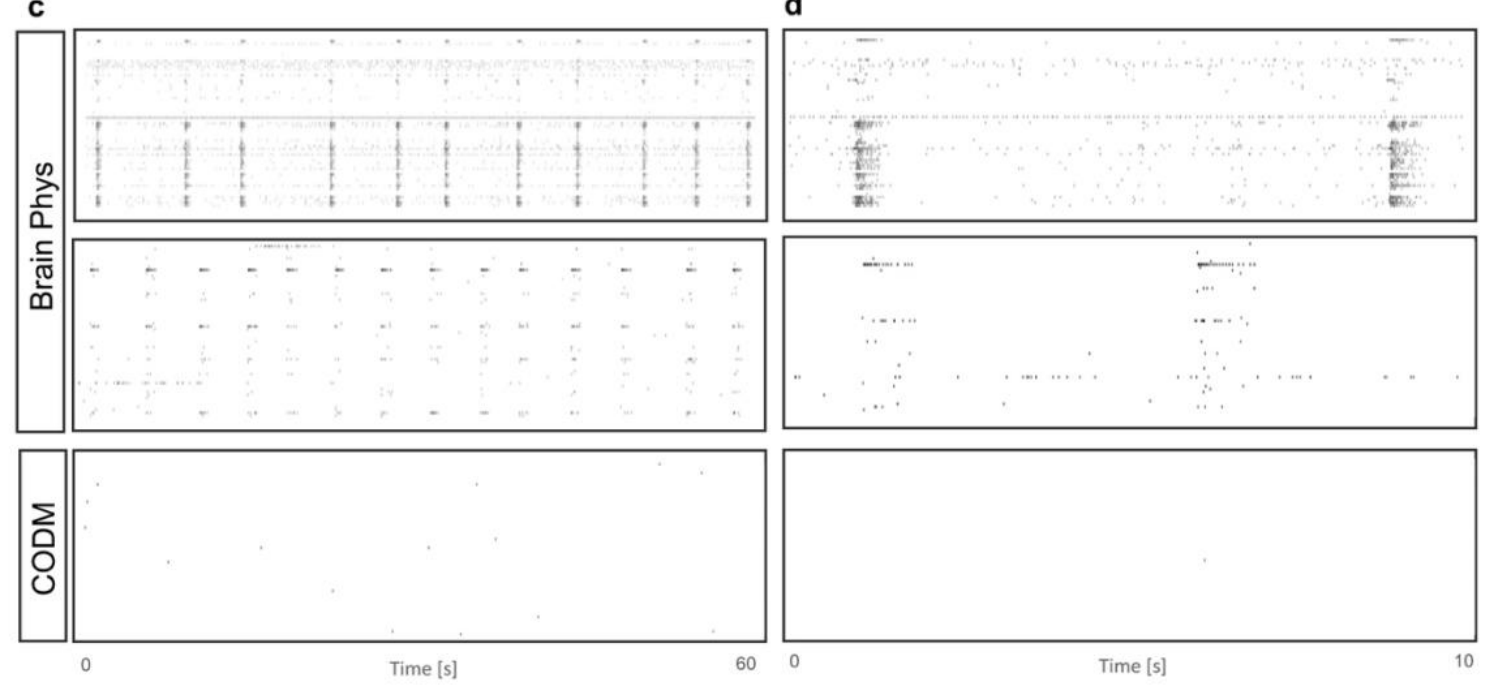

e

Whole MEA Average
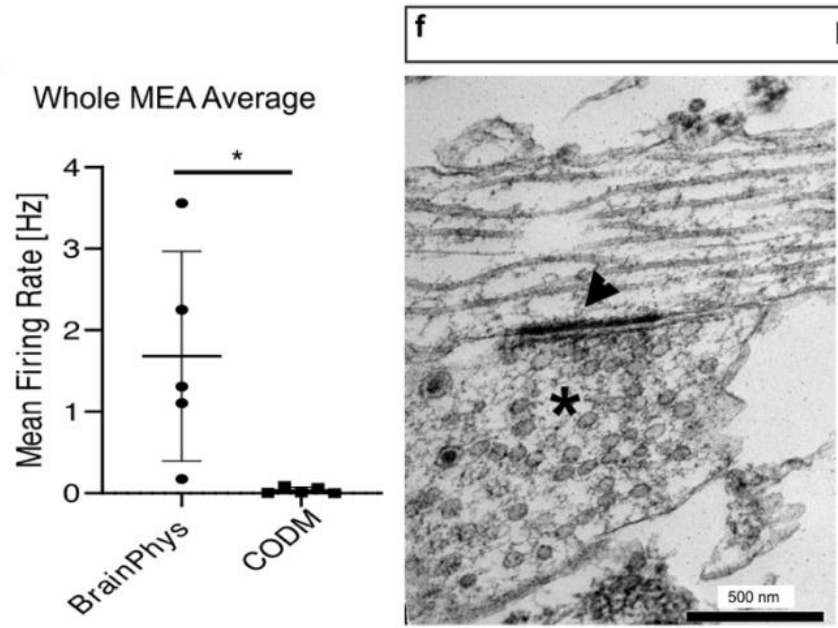

Brain Phys

\section{Supplementary Figure 5.}

975 a, b. Representative bright field and immunohistochemistry images showing brain Phys and CODM 976 cultured cortical organoids transduced with AAV9.SYN.mcherry viral vectors. c, d. Representative 977 spike raster plots showing firing patterns of organoids across all electrodes with marked network 978 bursts in Brain Phys, but not in CODM cultured organoids. e. whole MEA mean firing rate activity 979 (mean $\pm \mathrm{SD}$, paired $t$ test, ${ }^{*} \mathrm{p}<0.01$ ). f. Ultrastructure electron microscopy images of Brain Phys cultured 980 cortical organoid showing synaptic clefts (head arrows) and synaptic vesicles (asterisks). Scale bars, $981250 \mu \mathrm{m}(\mathrm{a}, \mathrm{b})$ 

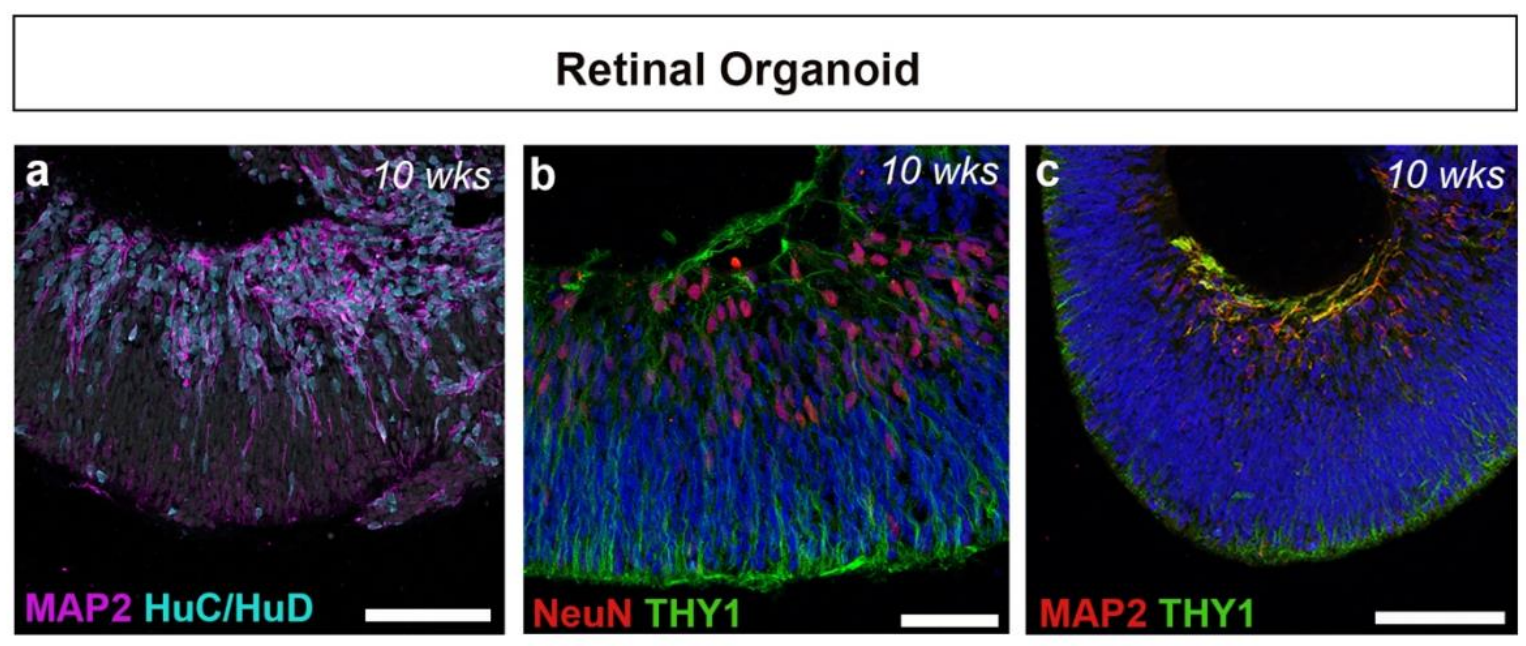

\section{Brain Organoid}
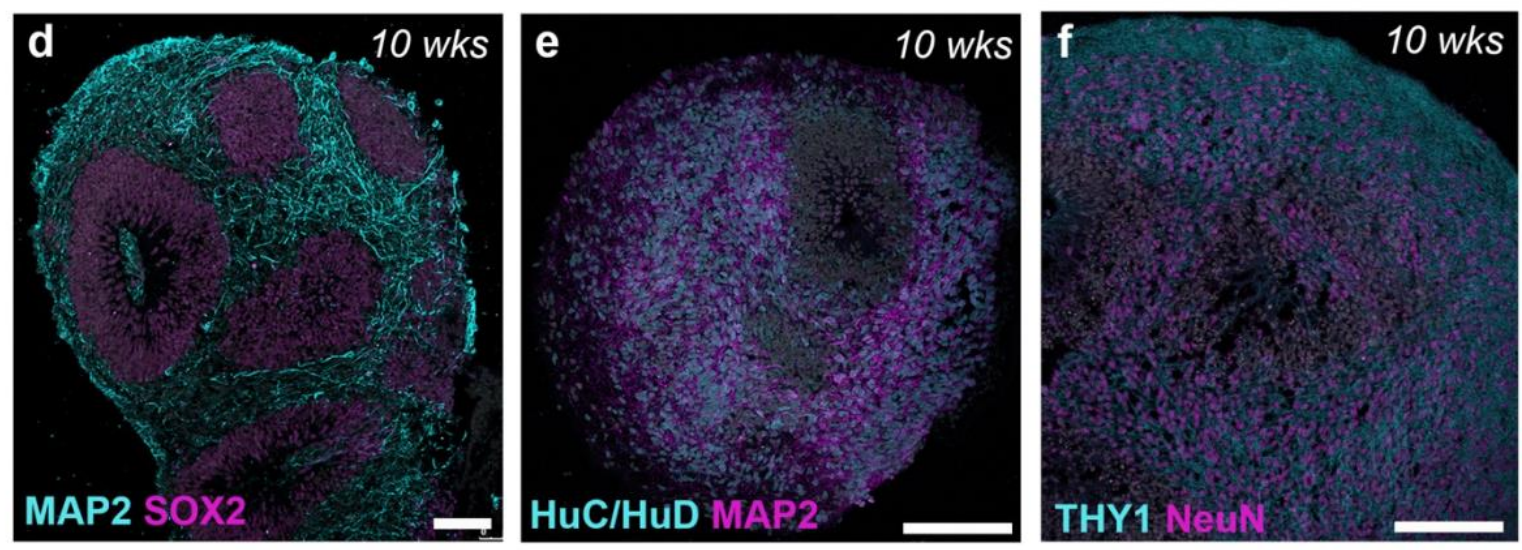

982

983

984

985

986

987

988

989

990

991

992

993

994

995

996

997

998

999

1000

Supplementary Figure 6. Neuronal markers on both retinal and brain organoids. a-f. Representative immunohistochemistry images from 10 weeks old retinal and cortical brain retinal organoids. a-c. Typical interneurons and retinal ganglion cell cells markers in the basal layer of the retinal organoid. d-f. The same markers are also expressed in cortical neurons of brain organoids. Scale bars, $75 \mu \mathrm{m}$ (d), $150 \mu \mathrm{m}$ (a, b, c, e, f).

\section{Supplementary Movies Legends}

\section{SMovie 1. Differentiation of early cortical progenitors}

Video of a typical representative day 35 cortical organoid showing neuroepithelium regions positive for PAX6 (red) and NESTIN (green). DAPI stained in blue.

\section{SMovie 2. Differentiation of neurons and glial cells}

Lightsheet video of a cleared whole cortical organoid at day 75 in culture. Organoid showing numerous neurons positive for CALRETININ (red) and glial cells positive for SB100 (green). DAPI stained in blue.

SMovie 3. Optic nerve-like formation between retinal and cortical organoids 
1001 Lightsheet video of cleared retinal-brain complex organoids. Video showing 510 z stacks starting at the retinal organoid with typical thick neuroepithelia showing MAP2 positive retinal ganglion cells and axonal projections leading towards cortical organoid with neurons positive 1004 for MAP2.

\begin{tabular}{|c|c|c|c|}
\hline \multicolumn{4}{|c|}{ Supplementary Table 1. Antibodies used for immunohistochemistry } \\
\hline Antigen & Host species & $\begin{array}{c}\text { Concentration } \\
\text { used }\end{array}$ & Supplier \\
\hline Calretinin & mouse & 1 in 200 & Merck Millipore (MAB1568) \\
\hline Caspase3 & rabbit & 1 in 200 & Abcam (ab2302) \\
\hline Ctip2 & rat & 1 in 200 & Abcam (Ab18465) \\
\hline FoxG1 & rabbit & 1 in 200 & Abcam (Ab18259) \\
\hline GABA & rabbit & 1 in 200 & Sigma (A2052) \\
\hline GFAP & rat & 1 in 200 & Sigma (345860) \\
\hline Ki67 & rabbit & 1 in 200 & Abcam (ab15580) \\
\hline MAP2 & chicken & 1 in 1000 & Abcam (ab5392) \\
\hline MAP2 & mouse & 1 in 200 & Merck Millipore (MAB3418) \\
\hline NCAD & mouse & 1 in 200 & BD Bioscience (610921) \\
\hline Nestin & mouse & 1 in 200 & Merck Millipore (MAB5326) \\
\hline Pax6 & rabbit & 1 in 200 & Bioscience (901302) \\
\hline PSD95 & goat & 1 in 200 & Abcam (ab12093) \\
\hline Satb2 & rabbit & 1 in 200 & Abcam (Ab34735) \\
\hline SB100 & mouse & 1 in 200 & Sigma (S2532) \\
\hline Sox2 & rabbit & 1 in 200 & Abcam (ab97959) \\
\hline Sox2 & mouse & 1 in 200 & BD (561469) \\
\hline Synaptophysin & mouse & 1 in 200 & Abcam (ab8049) \\
\hline TBR1 & rabbit & 1 in 200 & Abcam (ab31940) \\
\hline Tuj1 & mouse & 1 in 2000 & $\begin{array}{l}\text { Thermo Fisher Scientific } \\
\text { (MA1118X) }\end{array}$ \\
\hline Vglut1 & Guinea pig & 1 in 200 & Merck Millipore (AB5905) \\
\hline
\end{tabular}

